\title{
1 Association mapping identified novel candidate loci affecting wood formation in Norway
}

2 spruce

3 John Baison ${ }^{1}$, Amarylis Vidalis ${ }^{3}$, Linghua Zhou ${ }^{1}$, Zhi-Qiang Chen ${ }^{1}$, Zitong Li ${ }^{9}$, Mikko J.

4 Sillanpää ${ }^{10}$, Carolina Bernhardsson ${ }^{1,2}$, Douglas Scoffield ${ }^{5}$, Nils Forsberg ${ }^{1}$, Lars Olsson ${ }^{8}$, Bo

5 Karlsson $^{11}$, Harry $\mathrm{Wu}^{1}$, Pär K. Ingvarsson ${ }^{4}$, Sven-Olof Lundqvist ${ }^{8,12}$, Totte Niittylä ${ }^{1}$, M

6 Rosario García-Gil ${ }^{1 *}$

$7 \quad *$ Double last authorship.

8 Corresponding authors: m.rosario.garcia@slu.se; john.baison@slu.se

9 Telephone Number: +46 (0) 907868413 Swedish University of Agricultural Science, Umeå, Sweden

2. Department of Ecology and Environmental Science, Umeå University, Umeå, Sweden

3. Section of Population Epigenetics and Epigenomics, Center of Life and Food Sciences Weihenstephan, Technische Universität München, München, Germany

4. Department of Plant Physiology, Umeå Plant Science Centre, Umeå University, Umeå, Sweden

5. Uppsala Multidisciplinary Center for Advanced Computational Science, Uppsala University, Uppsala, Sweden

6. Department of Ecology and Genetics: Evolutionary Biology, Uppsala University, Uppsala, Sweden

7. Department of Plant Biology, Uppsala BioCenter, Swedish University of Agricultural Science, Uppsala, Sweden.

8. RISE Bioeconomy, Drottning Kristinas väg 61, SE-114 86 Stockholm, Sweden 
31 Total Word Count: 6500

32 Introduction Word Count: 953

33 Materials and methods Word Count: 1930

34 Results Word Count: 1565

35 Discussion Word Count: 1917

36 Conclusion Word Count: 91

37 Acknowledgements Word Count: 48

\section{Figures}

Fig. 1 Outline of the association mapping approach (Colour)

Page 9

$41 \quad$ Fig 2. Phenotype trajectories (Colour)

\section{Tables}

47 Table 1 List of the phenotypes, their abbreviations and measurement unit Page 8 


\section{Supplementary Data}

$51 \quad$ Fig.S1 Phenotype Trajectories (Colour)

Page 1

52 Table S1 ConGenIE BLAST search of contigs with significant QTLs

Page 10

53 Methods S1 PVE evaluation of a QTL

Page 33

54 Methods S2 Association mapping script

Page 33

Methods S3 Stabilization selection script

Page 34

\section{Summary}

57 studies and support current Norway spruce breeding efforts. 
Introduction

77 Norway spruce (Picea abies (L.) Karst.) is a dominant boreal softwood species of significant economic and ecological importance (Hannrup et al., 2004). Long-term Norway spruce breeding programmes for improvement of growth and survival were initiated in the 1940s and recently, wood quality has become one of the priority traits (Bertaud \& Holmbom, 2004;

81 Hannrup et al., 2004). Norway spruce breeding in Sweden complete one cycle in about 20 years and such long generation times make improvements in growth and wood quality very slow. Among wood quality traits, wood density is considered a key indicator of stability, strength and stiffness of sawn timber (Hauksson et al., 2001). Several studies of wood quality observed that fast growth conflicts with high quality wood, as shown by the negative genetic correlation between wood volume growth and density in Norway spruce ( Olesen, 1977;

87 Dutilleul et al., 1998; Chen et al., 2014). In order to combine fast growth and desirable wood properties through breeding, and to shorten the breeding cycle, it is therefore imperative to design effective early selection methods and breeding strategies. In an effort to design optimal breeding and selection strategies for reducing or breaking negative genetic correlations

91 between traits it is essential to identify alleles that are responsible for generating favourable or

92 unfavourable genetic correlations (Hallingbäck et al., 2014).

93 When DNA markers were first introduced in 1980s, tree breeders were provided a 94 possibility to correlate phenotypes with polymorphic DNA markers and to conduct selection 95 using genotypes instead of phenotypes (Lande \& Thompson, 1990). Groover et al. (1994) first 96 identified quantitative trait loci (QTL) for wood density variation in loblolly pine using 97 linkage analyses based on segregating family pedigrees. However, maker-aided selection 98 (MAS) based on results from QTL analyses was never implemented in practical tree breeding 99 due to the so-called Beavis effect (e.g. inflated estimates of allelic effects and underestimation 
100 of QTL number for economically important traits) (Beavis, 1998), inconsistent associations

101 among different families and the low transferability of markers (Strauss et al., 1992).

102 Association Mapping (AM) is a more powerful QTL detection method that was introduced to

103 tree genetics using a candidate gene approach (Thumma et al., 2010). AM overcomes the

104 limited resolution of family-based QTL mapping by relying on historical recombination in the

105 mapping population ( Neale \& Savolainen, 2004; Thavamanikumar et al., 2013; Huang \&

106 Han, 2014). The effectiveness of AM relies on genome-wide levels of LD, which decays

107 rapidly within coding regions in conifer species, however, it may be extensive in certain non-

108 coding regions (Moritsuka et al., 2012). Fast-decaying LD, coupled with complex polygenic

109 nature for both growth and wood quality traits (Hall et al., 2016) implies that a large number

110 of genomic regions need to be investigated to identify significant QTL (Beaulieu et al., 2011).

111 The availability of a draft genome sequence for Norway spruce (Nystedt et al., 2013)

112 has opened new possibilities for the development of genetic markers to conduct both AM at

113 the genome-wide level (genome-wide association, GWAS) and genomic selection (GS).

114 Several reduced representation-based approaches such as sequence capture and transcriptome

115 sequencing (Hirsch et al., 2014) have been developed as complexity-reduction methods suited

116 for studying large genomes, such as the 20Gb Norway spruce genome. These approaches

117 reduce the sequence space by decreasing the repetitive sequence content of the genome. In

118 this study we employed a solution-based sequence capture method.

119 Several AM studies have been performed in trees and have identified genetic loci

120 linked to, for instance, wood properties in Populus trichocarpa (Porth et al., 2013), adaptive

121 traits in Pinus contorta (Parchman et al., 2012) and to wood quality traits in Eucalyptus

122 (Porth et al., 2013; Resende et al., 2012). Such studies aimed at dissecting the genetic basis of

123 wood properties can benefit from the application of mathematical functions that account for

124 year-to-year variation across annual growth rings, cambial age and distance from pith (Li et 
125 al., 2014). Mathematical modelling allows the incorporation of phenotypic growth trends that

126 increase the precision and resolution of QTL detection through the integration of the

127 phenotype information over multiple time points and reduction of residual variance (Ma et al.,

128 2002). Such functional mapping analysis can be conducted using a multistage approach

129 (Heuven \& Janss, 2010). First, the phenotype trends of each individual are modelled using

130 curve-fitting methods and the parameters describing the curve are then considered as latent

131 traits. The latent traits are then used in an independent association analyses to search for

132 genomic regions affecting the trait and to estimate genetic marker effects (Li et al., 2014).

133 In this study, we applied a functional genome-wide association mapping (AM)

134 approach to identify genomic regions contributing to wood quality traits in Norway spruce

135 [Picea abies (L.) Karst.]. Estimated breeding values (EBVs) were calculated for growth and

136 wood quality traits at the resolution of annual growth rings and were then used to extract

137 latent traits from fitting quadratic splines, Fig. 1a. We applied quadratic splines since

138 traditional analyses that utilise a single point data across annual growth rings may confound

139 the analyses by averaging across a full sample. Such averaging may obscure mechanisms

140 acting at specific time points during wood formation and will make identification of

141 underlying genes more difficult. In this study, we have refined our data in order to cover

142 within ring features in earlywood (EW) and latewood (LW), as well as in a more weather

143 influenced part in between named transitionwood (TW). This study has also performed the

144 first analysis of number of cells per ring calculated from SilviScan data. Penalized LASSO

145 regression (Tibshirani, 1996) and the stabilizing selection probability method of

146 (Meinshausen \& Bühlmann, 2010) were then used, Fig. 1c, to detect significant associations

147 between latent traits derived from EBVs and 178101 SNP markers covering the Norway

148 spruce genome, Fig. 1b.

149 


\section{Materials and Methods}

\section{$151 \quad$ Plant material and phenotype data}

152 Plant material and phenotype data used in this study have previously been described in Chen 153 et al. (2014). In brief, two progeny trails were established in 1990 in Southern Sweden 154 (S21F9021146 aka F1146 (trial1) and S21F9021147 aka F1147 (trial2)). These trials were 155 composed of 1373 and 1375 open pollinated families, respectively, and form the basis of our 156 analyses. We selected 517 families in 112 sampling stands to use in the investigation of wood 157 properties. At each site, increment wood cores of $12 \mathrm{~mm}$ were collected from six trees of the 158 selected families at breast height $(1.3 \mathrm{~m})(6$ progeny $\times 2$ sites $=12$ progenies in total $)$. A total 159 of 5618 trees, 2973 and 2645 trees from the F1146 and F1147 trails respectively, were

160 analysed. The pith to bark profiling of the wood physical attributes was analysed using the

161 SilviScan technology (Evans 1994, 2006) at Innventia, Stockholm, Sweden, where the initial 162 data evaluations were performed using customized methods. These methods focus on the 163 identification and dating of all annual rings and their compartments of earlywood, 164 transitionwood and latewood. For the current study, Innventia also calculated three additional 165 traits, number of cells per ring (NC), wood percentage (WP), and a trait named Mass Index 166 (MI), introduced to express the relative amount of biomass, all derived from the SilviScan 167 data. MI was then used to identify trees with an uncommon positive correlation between 168 density and growth, that is more biomass. The traits included in the current study are listed in 169 Table 1. 
176 Table 1 List of the phenotypes, their abbreviations and measurement unit.

\begin{tabular}{|c|c|c|}
\hline Phenotype & Abbreviation & Unit \\
\hline Ring wood density & WD & $\mathrm{kg} \mathrm{m}^{-3}$ \\
\hline Early wood density & EWD & $\mathrm{kg} \mathrm{m}^{-3}$ \\
\hline Transition wood density & TWD & $\mathrm{kg} \mathrm{m}^{-3}$ \\
\hline Latewood density & LWD & $\mathrm{kg} \mathrm{m}^{-3}$ \\
\hline Ring width & RW & $\mu \mathrm{m}$ \\
\hline Early wood ring width & ERW & $\mu \mathrm{m}$ \\
\hline Transition wood ring width & TRW & $\mu \mathrm{m}$ \\
\hline Latewood ring width & LRW & $\mu \mathrm{m}$ \\
\hline Ring number of cells & $\mathrm{NC}$ & \\
\hline Early wood number of cells & $\mathrm{ENC}$ & \\
\hline Transition wood number of cells & $\mathrm{TNC}$ & \\
\hline Latewood number of cells & LNC & \\
\hline Early wood percentage & $\mathrm{EP}$ & $\%$ \\
\hline Transition wood percentage & $\mathrm{TP}$ & $\%$ \\
\hline Latewood percentage & LP & $\%$ \\
\hline Early/Latewood percentage & EP/LP & $\%$ \\
\hline Modulus of elasticity & MOE & $\mathrm{GPa}$ \\
\hline Mass Index (Density x Growth) & MI & \\
\hline
\end{tabular}


bioRxiv preprint doi: https://doi.org/10.1101/292847; this version posted July 12,2018 . The copyright holder for this preprint (which was not certified by peer review) is the author/funder, who has granted bioRxiv a license to display the preprint in perpetuity. It is made available under aCC-BY 4.0 International license.

180 


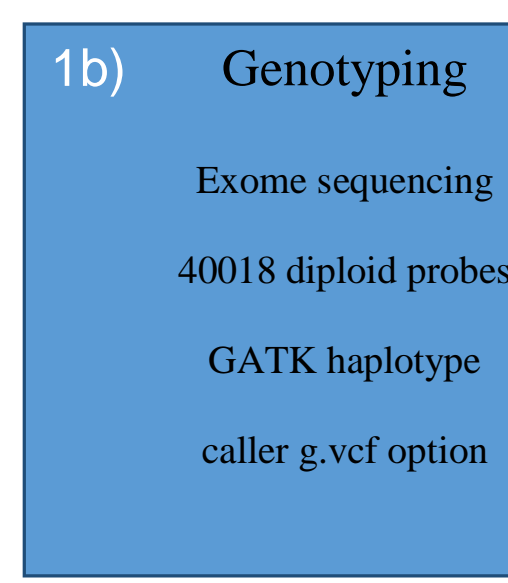

Fig 1. Outline of the association mapping approach: 1a) Mother Estimated breeding values (EBVs) were determined using a univariate, bivariate or multivariate mixed linear model based on the different fitness of the model with the resultant values adjusted with the mean. The adjusted EBVs were plotted against cambial age (annual ring number) to produce time trajectories for each trait. A quadratic spline curve model was then applied to the EBVs to estimate latent-traits. 1b) Sequence capture on the 517 from 40018 diploid probes resulted in 178101 single-nucleotide

\section{Candidate gene identification for future}

1d) functional genomics work

\section{1a) Phenotyping}

Mother EBVs at each (BLUP)

Curve Models to extract Latent-traits based on EBVs (BLUP)

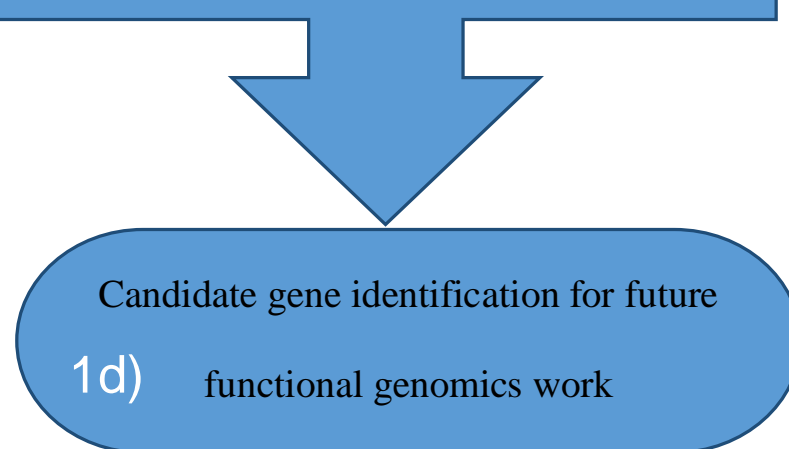


191 polymorphisms (SNPs). 1c) Association mapping was the performed using a multi-locus LASSO penalized regression method with PCA

192 components acting as covariates accounting for the population structure in our mother trees. 1d) Finally, a candidate gene identification process

193 for contigs with significant SNPs was conducted in ConGenIE and public sequence databases. 


\section{Statistical analysis}

196 EBVs were calculated for each cambial age (annual ring) separately and used for statistical

197 modelling to derive latent traits. The variance and covariance components were estimated

198 using ASREML 4.0 (Gilmour et al., 2014) as described in Chen et al., (2014). In brief, the

199 EBVs at each cambial age were estimated using a univariate, bivariate or multivariate mixed

200 linear models. The fit of different models were evaluated using the Akaike Information

201 Criteria (AIC) and the optimal model was selected based on a compromise of model fit and

202 complexity. Breeding values were then centred in order to obtain within genotype trends. A univariate linear mixed model for joint-site analysis was implemented as:

where $\mathrm{Y}_{\mathrm{ijkl}}$ is the observation on the $l$ th tree from the $k$ th family in jth block within the

$i$ th site, $u$ is the general mean, $\mathrm{S}_{\mathrm{i}}$ and $\mathrm{B}_{\mathrm{j}(\mathrm{i})}$ are the fixed effects of the $i$ th site and the $j$ th block

within the ith site, respectively, $\mathrm{F}_{\mathrm{k}}$ and $\mathrm{SF}_{\mathrm{ik}}$ are the random effects of the $k$ th family and the

random interactive effect of the $i$ th site and $k$ th family, respectively, $e_{i j k l}$ is the random

residual effect. Multivariate mixed linear models were used to estimate BV for different

211 phenotype traits if the model fitted better than bivariate or univariate based on AIC.

212 A number of trees were observed that broke the negative correlation usually observed between density and growth. These trees exhibited both high density and fast growth, thus

214 larger biomass. In order to identify putative genes involved in this favourable combination of 215 traits, we defined a new trait termed Mass Index (MI), that we subsequently used in the 216 association mapping. The MI was defined as follows: Mass index $=($ Individual average density/population average density) $*$ (individual 

individuals with an index $>1$ indicate a wood mass per length unit than the population average in the cross-section at breast height. The index was calculated for all progeny and

222 were used to calculate BVs for the 517 mother trees.

The EBVs were plotted against cambial age (annual ring number) to produce time trajectories for each trait (Fig. 2 and Fig. S1) and used to estimate latent curve parameters. At the first stage, all the trajectories versus cambial age were fitted with a quadratic spline with multiple knots in order to describe the dynamics of the EBVs across age. In this study, this was done with the values of four parameters obtained from the spline fitting: the intercept, the slope and two knot parameters (K1 and K2). The intercept and slopes were used to evaluate the mean and rate of change for the trait across the annual rings, respectively. $\mathrm{K} 1$ and $\mathrm{K} 2$ represent inflection points in the cambial age trajectories where the development of the EBVs enters new phases. These two points (K1 and K2) are therefore supposed to have biological significance, warranting a closer analysis of the genes imparting these shifts in the EBVs dynamics. The four latent traits show lower correlations compared to the direct measurements on the original scales and they also have constant variances, thereby reducing the need to account for residual dependencies in the model (Li et al., 2014).

The general definition of a quadratic spline with multiple knots is as follows:

$\beta(t)=b_{0}+b_{1} t+b_{2} t^{2}++b_{3}\left(t-t_{t}\right)^{2}+b_{4}\left(t-t_{2}\right)^{2}+\ldots+b_{2+k}\left(t-t_{k}\right)^{2}$

which is continuous and where $t_{\mathrm{i}}\left(i=1, \ldots, k ; t_{1}<t_{2} \ldots<t_{k}\right)$ are defined as knots, and $\left(t-t_{i}\right)^{2}{ }_{+}=(t$ $\left.-t_{i}\right)^{2}$ if $t>t_{i}\left(t_{i}>0 ; i=1, \ldots, k\right)$, and otherwise is equal to zero. The number of knots has to be properly defined in order to provide an accurate description of the data under investigation, as well as functional starting points for the search of their locations (Li et al., 2015). In our case, 
242 since the growth pattern of wood property traits were not complex, we choose two knots of

243 the time interval.

244 Hence, the quadratic spline model to describe the growth trajectory of individual $i$

245 applied in this study was defined as:

$246 \quad y_{i}(t)=\beta_{0}+\beta_{1} t+\beta_{2}\left(t-t_{1}\right)_{+}+\beta_{3}\left(t-t_{2}\right)_{+}+\varepsilon_{i}(t), \quad \varepsilon_{i}(t) \stackrel{\text { i.i.j. }}{\square} N\left(0, \sigma^{2}\right)$.

247 Then the intercept $\beta_{0}$, slope $\beta_{1}, \beta_{2}$, (Knot $\left.1(\mathrm{k} 1)\right)$ and $\beta_{3}($ Knot $2(\mathrm{k} 2))$ are estimated by 248 standard least squares, and their estimates were considered as the latent trait in the subsequent

249 QTL analysis conducted in R-studio (Team, 2015). The latent traits were then analysed using

250 the LASSO model in order to identify SNPs showing significant associations to the traits.

251

252

253

254

255

256

257

A)

\section{Density}

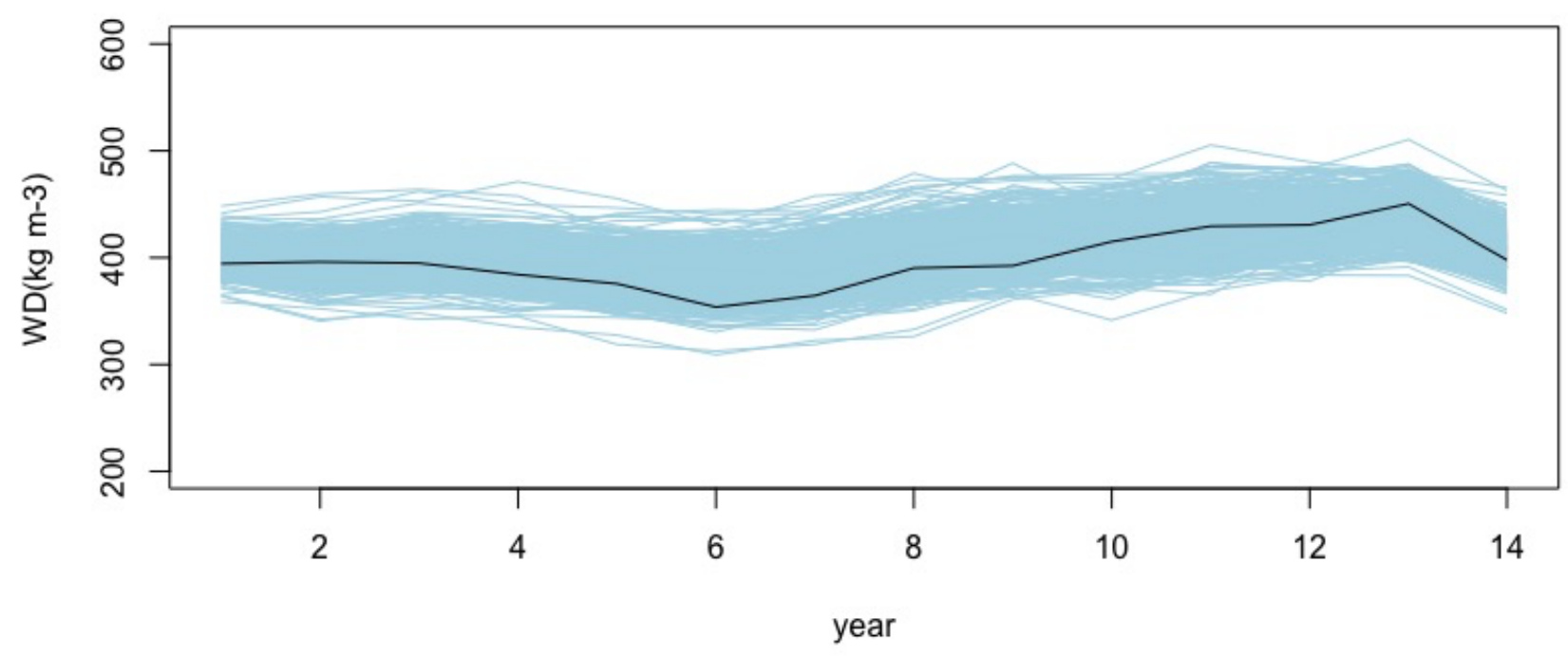


bioRxiv preprint doi: https://doi.org/10.1101/292847; this version posted July 12,2018 . The copyright holder for this preprint (which was not certified by peer review) is the author/funder, who has granted bioRxiv a license to display the preprint in perpetuity. It is made available under aCC-BY 4.0 International license.

259 B)

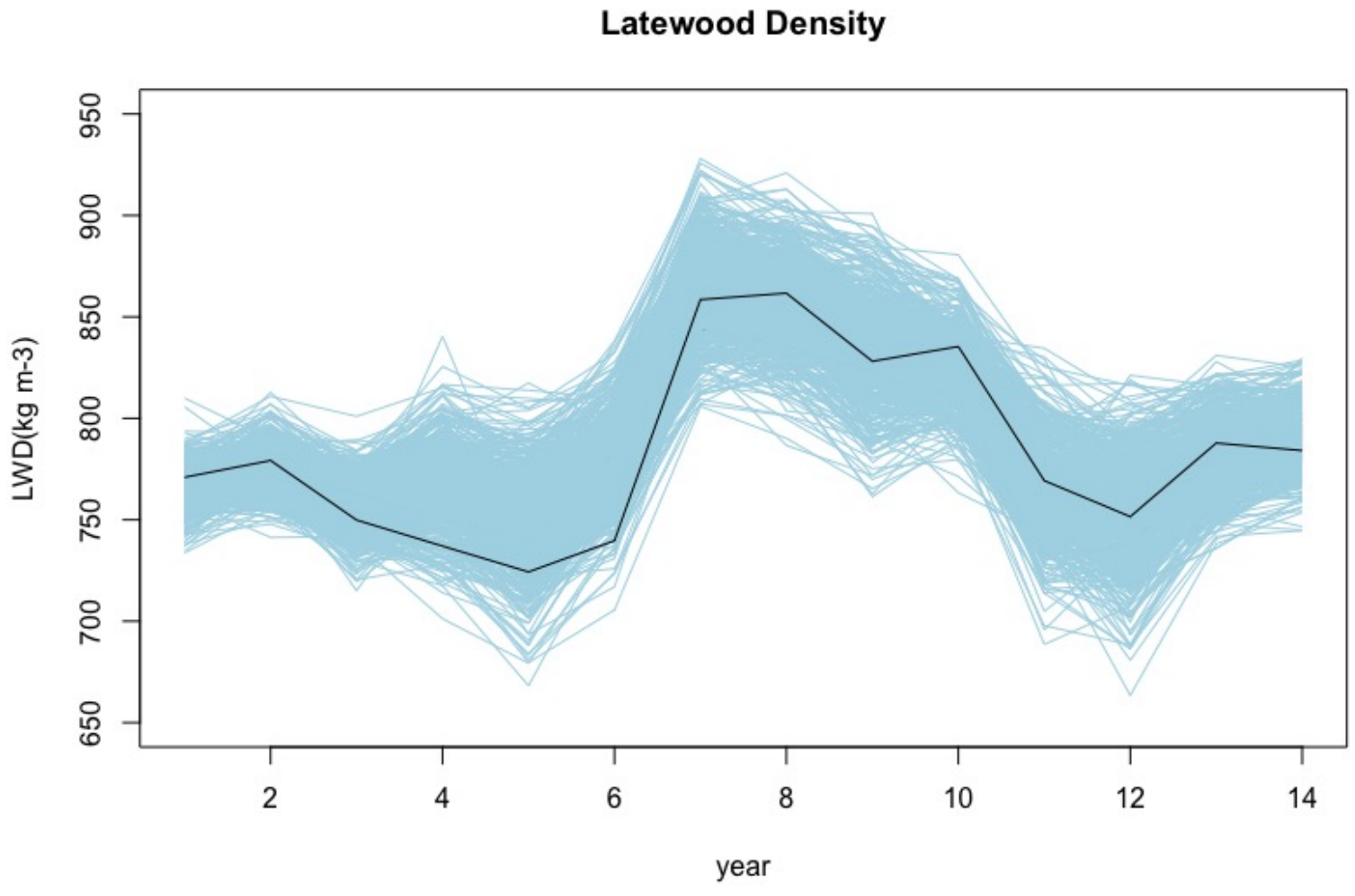

261

262 C)

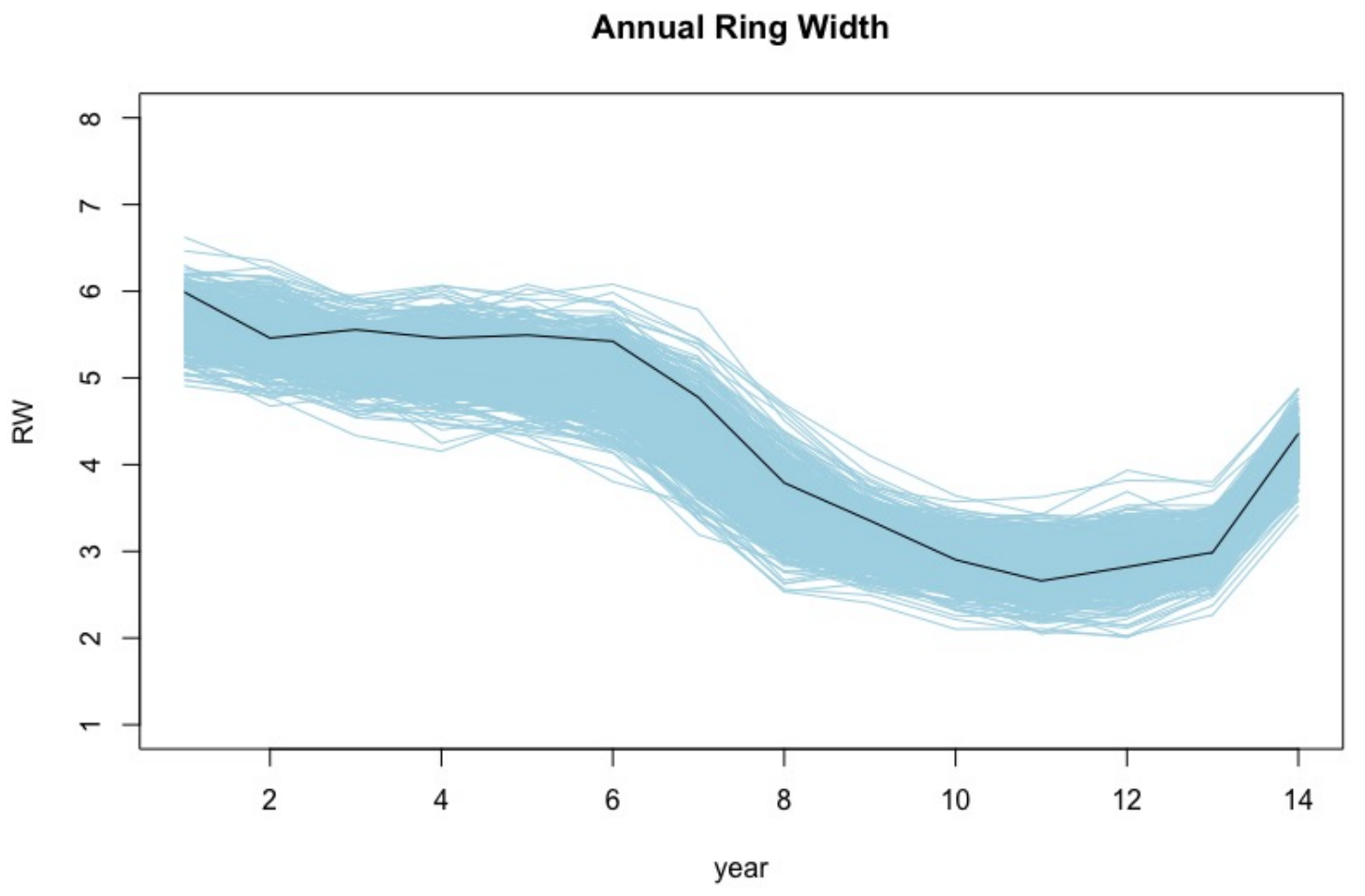




\section{Latewood Ring Width}

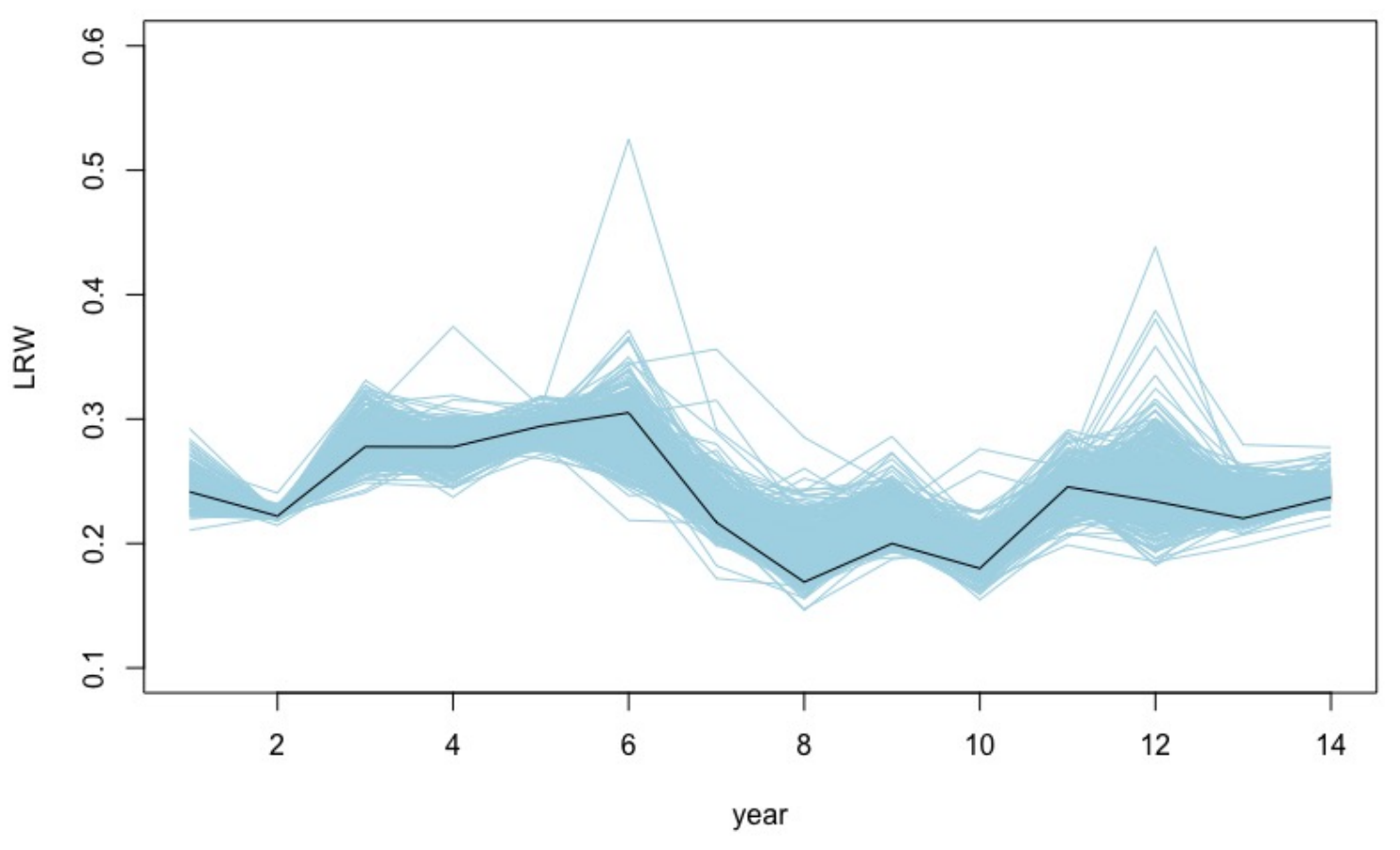

267 Fig 2. EBV trajectories of four wood quality traits by time:(A) wood density, (B) latewood

268 density, (C) annual ring width and (D) latewood ring width. Individual trajectories for each trait are shown in light blue lines and the black line represents the mean trajectory for the phenotype. These individual trajectories were used to determine the four latent traits of each tree, using quadratic splines with two knots.

274 Total genomic DNA was extracted from 517 unrelated individuals using the Qiagen Plant

275 DNA extraction protocol with DNA quantification performed using the Qubit ${ }^{\circledR}$ ds DNA 276 Broad Range (BR) Assay Kit (Oregon, USA). Sequence capture was performed using the 40

277018 diploid probes previously designed and evaluated for P. abies (Vidalis et al., 2018) and 278 samples were sequenced to an average depth of 15x using an Illumina HiSeq 2500 (San 
279 Diego, USA). Raw reads were mapped against the P.abies reference genome v1.0 using

280 BWA-mem (Langmead \& Salzberg, 2012; Li \& Durbin, 2009). SAMTools v.1.2 (Li et al.,

281 2009) and Picard v.1.140 (McKenna et al., 2010) were used for sorting and removal of PCR

282 duplicates. Variant calling was performed using GATK HaplotypeCaller v.3.6 (McKenna et

283 al., 2010) in gVCF output format. Samples were then merged into batches of $\sim 200$ before all

284517 samples were jointly called.

285 Variant Quality Score Recalibration (VQSR) method was performed in order to avoid

286 the use of hard filtering for exome/sequence capture data. For the VQSR analysis two datasets

287 were created, a training subset and input file. The training dataset was derived from a Norway

288 spruce genetic mapping population with loci showing expected segregation patterns

289 (Bernhardsson et al., 2018) and assigned a prior value of 15.0. The input file was derived

290 from the raw sequence data using GATK best practices with the following parameters:

291 extended probe coordinates by +100 excluding INDELS, excluding LowQual sites, and

292 keeping only bi-allelic sites. The following annotation parameters QualByDepth (QD),

293 MappingQuality (MQ) and BaseQRankSum, with tranches 100, 99.9, 99.0 and 90.0 were then

294 applied for the determination of the good versus bad variant annotation profiles. After

295 obtaining the variant annotation profiles, the recalibration was then applied to filter the raw

296 variants. Using VCFTools v.0.1.13 (Danecek et al., 2011), SNP trimming and cleaning

297 involved the removal of any SNP with a minor allele frequency (MAF) and "missingness" of

$298<0.05$ and $>20 \%$, respectively.

299 The resultant SNPs were annotated using default parameters for snpEff 4 (Cingolani et

$300 a l ., 2012)$. Ensembl general feature format (GTF, gene sets) information was utilized to build

301 the $P$. abies snpEff database.

302

303 Genetic Structure 
304 A principal component analysis (PCA) was performed on the sampled trees using SNPs

305 derived from the sequence capture data. SNPs with missing values following VQSR were

306 imputed using the nearest neighbour principle in TASSEL (Bradbury et al., 2007). This

307 approach was essential considering that PCA demands no missing data points. The covariate

308 matrix derived from the PCA was then displayed by plotting principal component 1 scores

309 against principal component 2 scores in Figure 2. The PCA plot was used to make inference

310 about the population structure. The first two components of the PCA covariate matrix

311 explaining most of the variation were then applied to the AM to account for population

312 structure and correcting for any stratification within the study.

313 Linkage disequilibrium was calculated using VCFtools v.0.1.13 software using the

314 squared correlation coefficient between genotypes (r2) within scaffolds using the "geno-r2".

315 The trend-line of LD decay with physical distance was fitted using nonlinear regression (Hill

$316 \&$ Weir, 1988) and the regression line was displayed using R (Team, 2015).

Trait Association Mapping

319 The LASSO model as described by Li et al (2014), Fig. 1c, was applied to all latent traits for 320 the detection of QTLs.

The LASSO model:

$$
\min _{\left(\alpha_{0}, \alpha_{j}\right)} \frac{1}{2 n} \sum_{i=1}^{n}\left(y_{i}-\alpha_{0}-\sum_{j=1}^{p} x_{i j} \alpha_{j}\right)^{2}+\lambda \sum_{j=1}^{p}\left|\alpha_{j}\right|
$$

where $y_{i}$ is the phenotypic value of an individual $i(i=1, \ldots, n ; n$ is the total number of individuals) for the latent trait $\beta_{0}, \beta_{1}, \beta_{2}$ or $\beta_{3}, \alpha_{0}$ is the population mean parameter, $x_{i j}$ is the genotypic value of individual $i$ and marker $j$ coded as 0,1 and 2 for three marker genotypes $\mathrm{AA}, \mathrm{AB}$ and $\mathrm{BB}$, respectively, $\alpha_{j}$ is the effect of marker $j(i=1, \ldots, n ; n$ is the total number of markers), and $\lambda(>0)$ is a shrinkage tuning parameter. A fundamental idea of LASSO is to 
328 utilize the penalty function to shrink the SNP effects toward zero, and only keep a small

329 number of important SNPs which are highly associated with the trait in the model.

330 The stability selection probability (SSP) of each SNP being selected to the model was

331 applied as a way to control the false discovery rate and determine significant SNPs (Gao et

332 al., 2014; Li \& Sillanpää, 2015). For a marker to be declared significant, a SSP inclusion ratio

333 (Frequency) was used with an inclusion frequency of at least 0.52 for all traits. This frequency

334 inferred that the expected number of falsely selected markers was less than one (1), according

335 to the formula of Buhlmann et al, (2014). Population structure was accounted for in all

336 analyses by including the first two principal components based on the genotype data as

337 covariates into the model. An adaptive LASSO approach (Zou et al. 2006) was used to

338 determine the percentage of phenotypic variance (PVE) $\left(H^{2}{ }_{Q T}\right)$ of all the QTLs (Methods S1).

339 These analysis were all performed in $\mathrm{R}$ (Team, 2015), with all the scripts provided in the

340 supplementary material.

\section{Candidate gene mining}

343 To assess homology of contigs with significant associations, a BLAST search was performed

344 against ConGenIE and public sequence databases, Fig. 1d. After the identification of

345 significant SNPs, the complete $P$. abies contigs that harboured the QTLs were then BLASTed

346 against the ConGenIE database and if no significant hit were detected the whole contig was

347 then extracted. The complete contigs in fasta format were then used to perform a nucleotide

348 BLAST (Blastn) search using the option for only highly similar sequences (megablast) in the

349 National Center for Biotechnology Information (NCBI) nucleotide collection database

350 (https://blast.ncbi.nlm.nih.gov/Blast.cgi?). 
355 Results

356 Norway spruce SNP identification and mapping population structure

357 All of the 517 Norway spruce mother trees in the study were considered for variant detection

358 and an average of 1.5 million paired end reads were sequenced per individual for the 40019

359 exome capture probes. This resulted in the identification of 178101 high confidence SNPs. In

360 order to account for effects derived from population stratification we performed a PCA and

361 identified two separate main population groups as well as a number of individuals scattered in

362 between these two main groups. Nevertheless, the differences due to population structure

363 were small with the first two principal components cumulatively explaining only $2.18 \%$ of the

364 genetic variation observed (Fig. 3). LD was also determined between all the SNPs, within

365 contigs as well as within significant contigs only and LD decay across physical distance is

366 plotted in Fig 4.

367

368 


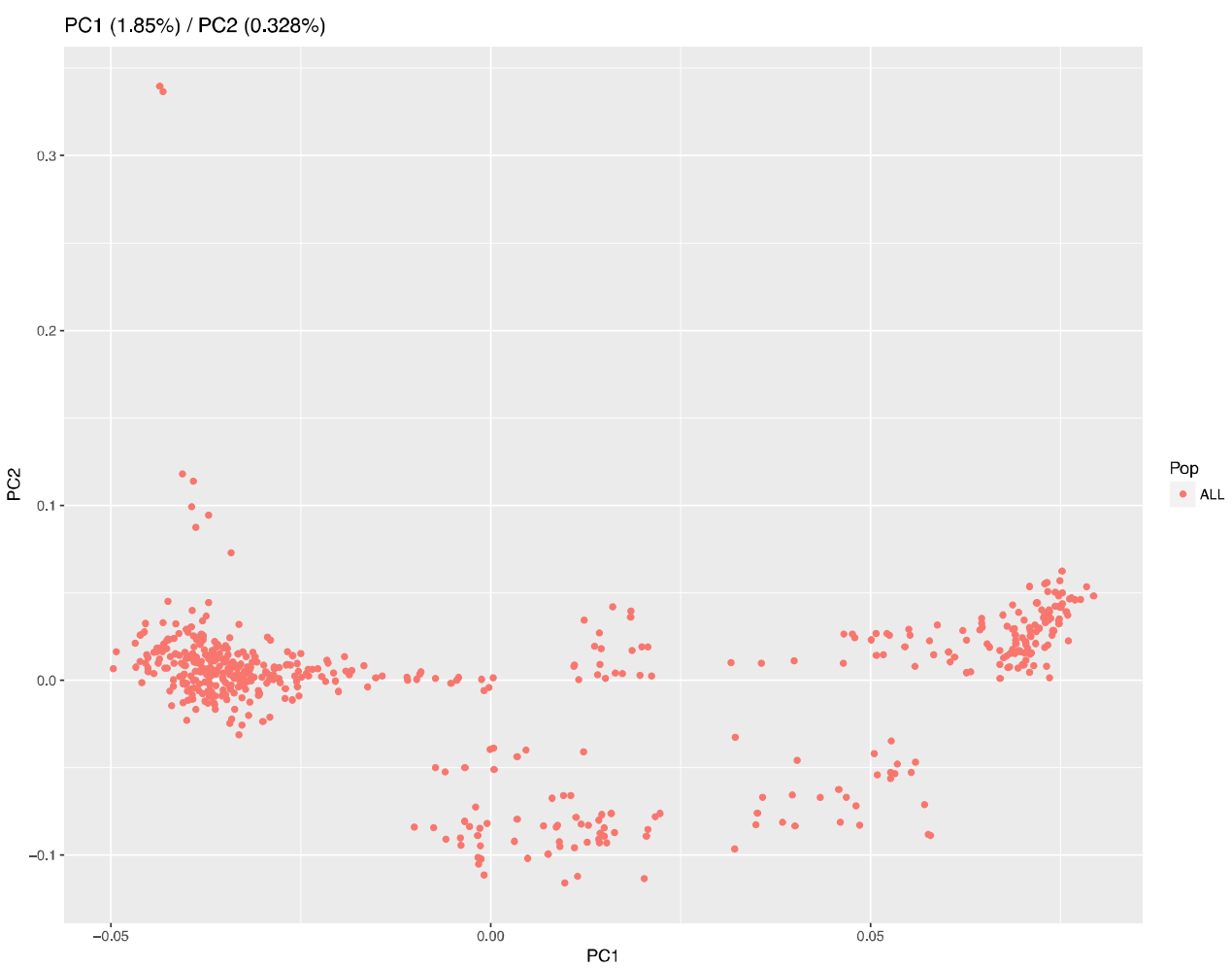

370

371 Fig 3. PCA plot of all the 517 mother trees. After VQSR and hard filtering of the SNPs,

372 imputation using the nearest neighbor principle was performed in TASSEL. The PCA 373 indicated a presence of two distinct populations within the 517 mother trees from the Norway 374 spruce breeding program in Sweden. The inferred population structure was used for the 375 correction of stratification within the AM analysis.

378 Employing a SSP inclusion frequency of at least 0.52 on the intercept, slope and two knots

379 (K1 and K2) as latent traits, we detected 51 significant QTL across 17 individual traits with 380 the phenotypic variances explained QTL $\left(H_{Q T L}^{2}\right)$ ranging from 0.01 to $4.93 \%$ (Table 2).

381 Several appreciable QTLs were identified with WD and RW having the highest number of 382 associations, at a total of 13 and 14 QTLs, respectively. This was followed by EP/LP-ratio, 383 which had six QTLs. WD, RW and EP/LP were the only three observed traits that have QTLs 
384 detected in all four latent traits. For these three phenotypes, the majority of the QTLs were

385 detected when the average ring phenotype was used to derive the latent traits (Table 2). NC

386 associated with one QTL that was detected for the entire ring, whilst six QTLs were identified

387 when EW, TW and LW were analysed separately, with $H^{2}$ QTL ranging from $0.01-4.93 \%$

388 (Table 2).

389

390

391

392

393

394

395

396

$397 \quad$ A)

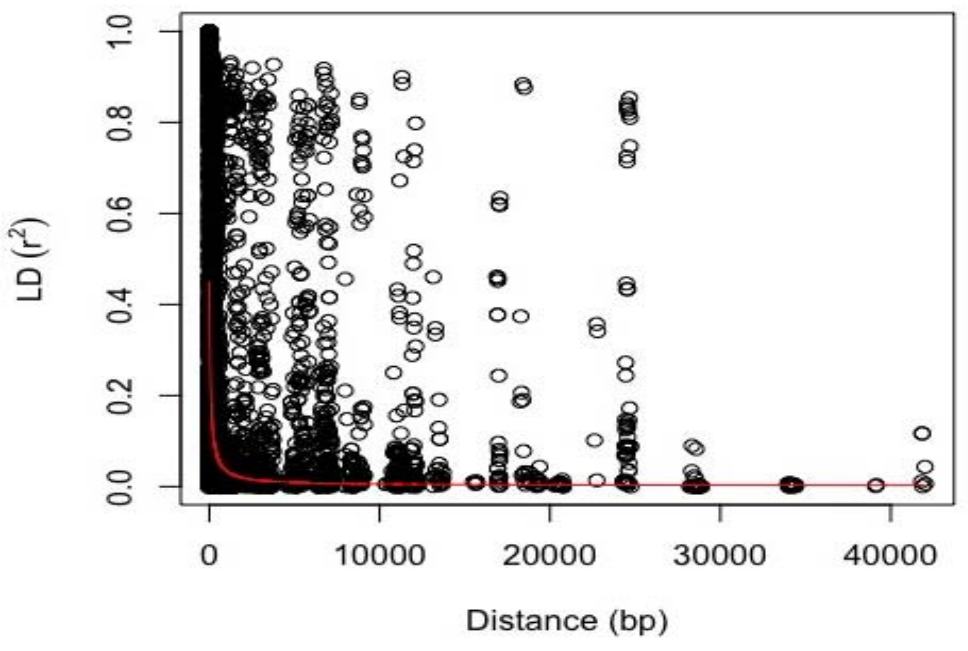


401

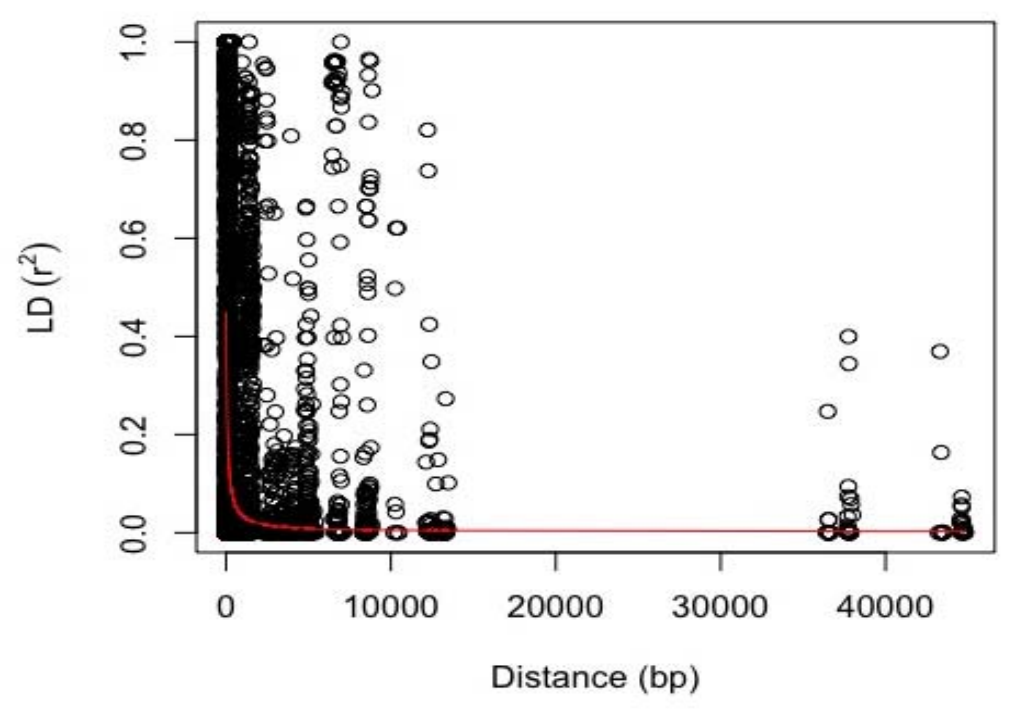

402

403

404

405

406 C)

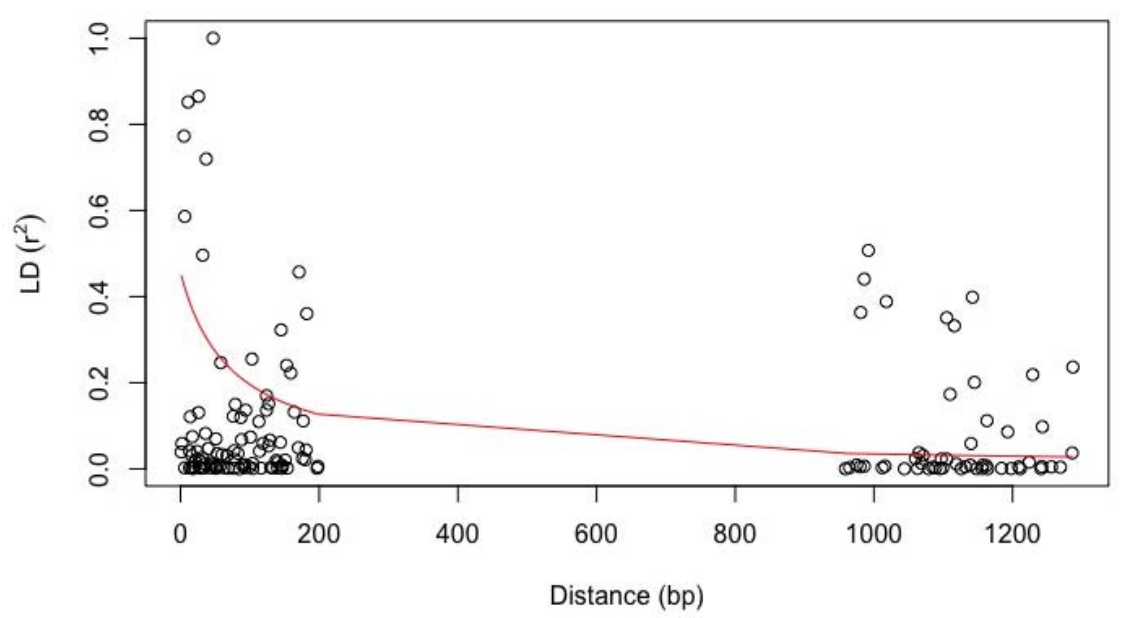

407

Fig 4. (A) Decay of linkage disequilibrium (LD) across all the tagged genomic sequences, the

409 majority being exoms. Squared coefficients of allele frequency $\left(r^{2}\right)$ are plotted against 
410 distance in base pairs. The fitted curve (red) is representative of the trend of decay from the

411 178101 SNPs utilised in the association mapping (AM). (B) Decay of LD with distance in

412 base pairs between sites from across 41 contigs with significant associations. (C) Decay of

413 LD across contig MA_96191 that has a significant association for ratio of percentage

414 earlywood vs latewood on which two probes were captured.

415

416

417 
419 Table 2 Phenotypes, Latent Traits, SNP, SNP feature, frequency and PVE

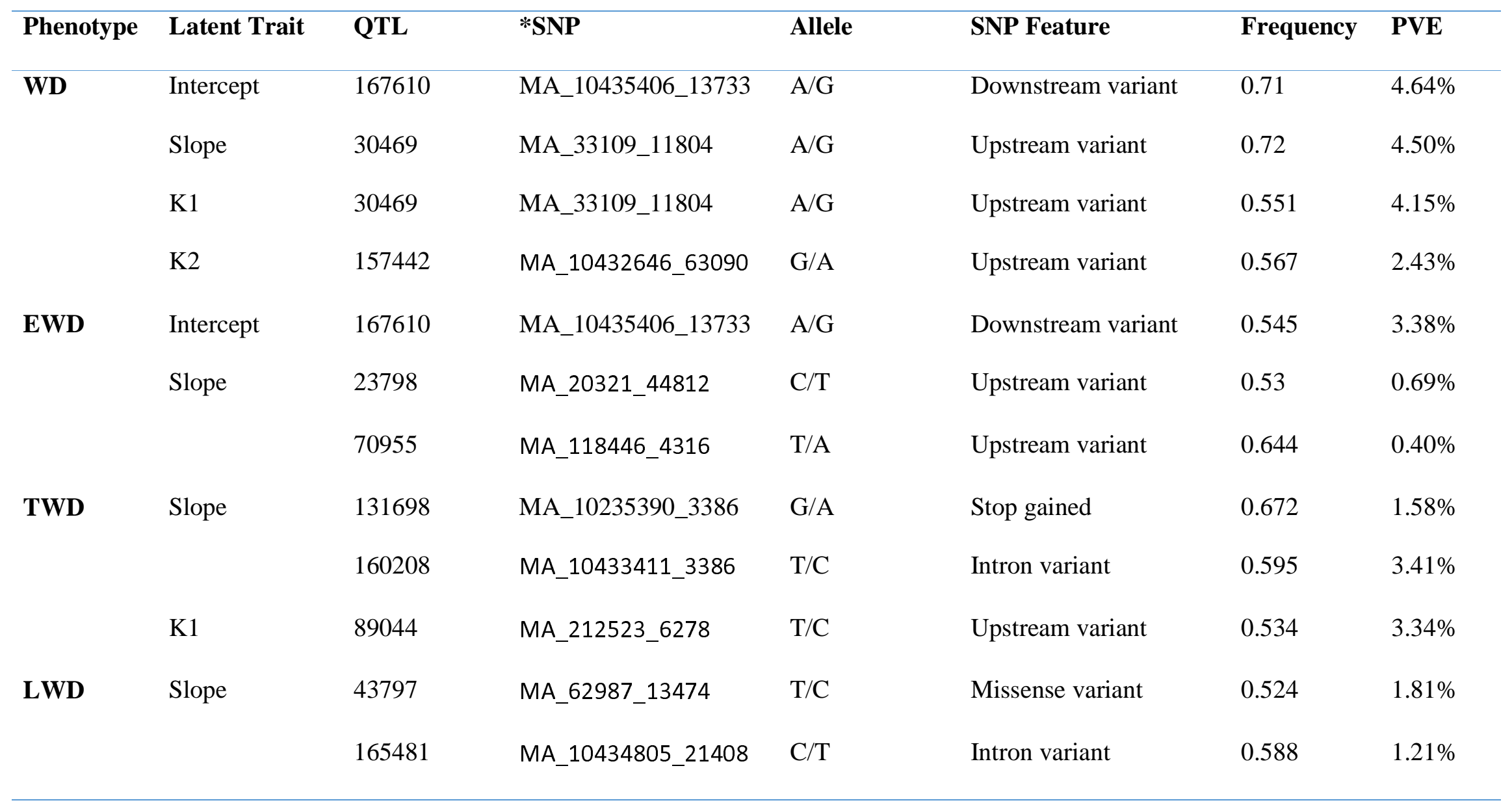




\begin{tabular}{|c|c|c|c|c|c|c|c|}
\hline & & 171223 & MA_10436058_4902 & G/A & Intron variant & 0.712 & $4.03 \%$ \\
\hline \multirow{4}{*}{$\mathbf{R W}$} & & 112394 & MA_879384_3894 & $\mathrm{C} / \mathrm{A}$ & Splice region variant & 0.692 & $2.56 \%$ \\
\hline & $\mathrm{K} 1$ & 23808 & MA_20322_28351 & $\mathrm{T} / \mathrm{G}$ & Synonymous variant & 0.554 & $1.78 \%$ \\
\hline & & 165481 & MA_10434805_21408 & $\mathrm{C} / \mathrm{T}$ & Intron variant & 0.533 & $0.18 \%$ \\
\hline & $\mathrm{K} 2$ & 23808 & MA_20322_28351 & $\mathrm{T} / \mathrm{G}$ & Synonymous variant & 0.55 & $1.20 \%$ \\
\hline \multirow{3}{*}{ TRW } & $\mathrm{K} 1$ & 33110 & MA_38472_13803 & $\mathrm{T} / \mathrm{A}$ & Upstream gene variant & 0.657 & $3.23 \%$ \\
\hline & & 89295 & MA_214776_1624 & G/A & Upstream gene variant & 0.688 & $4.51 \%$ \\
\hline & $\mathrm{K} 2$ & 111057 & MA_817099_1105 & $\mathrm{T} / \mathrm{A}$ & Missense variant & 0.672 & $1.20 \%$ \\
\hline \multirow[t]{2}{*}{ LRW } & Intercept & 143628 & MA_10428744_29330 & $\mathrm{C} / \mathrm{T}$ & Downstream variant & 0.668 & $0.5 \%$ \\
\hline & $\mathrm{K} 2$ & 164772 & MA_10434624_20686 & $\mathrm{C} / \mathrm{A}$ & Downstream variant & 0.571 & $0.06 \%$ \\
\hline
\end{tabular}




\begin{tabular}{|c|c|c|c|c|c|c|c|}
\hline MOE & Slope & 165481 & MA_10434805_21408 & $\mathrm{C} / \mathrm{T}$ & Intron variant & 0.602 & $1.00 \%$ \\
\hline ENC & & 167610 & MA_10435406_13733 & $\mathrm{A} / \mathrm{G}$ & Downstream variant & 0.685 & $0.01 \%$ \\
\hline TNC & & 126785 & MA_9447489_687 & $\mathrm{A} / \mathrm{C}$ & Upstream gene variant & 0.68 & $4.93 \%$ \\
\hline \multirow[t]{2}{*}{ LNC } & Intercept & 143628 & MA_10428744_29330 & $\mathrm{C} / \mathrm{T}$ & Downstream variant & 0.66 & $3.14 \%$ \\
\hline & Slope & 143628 & MA_10428744_29330 & $\mathrm{C} / \mathrm{T}$ & Downstream variant & 0.672 & $4.77 \%$ \\
\hline $\mathbf{T P}$ & & 132014 & MA_10251995_2442 & $\mathrm{A} / \mathrm{C}$ & Upstream gene variant & 0.601 & $3.22 \%$ \\
\hline $\mathbf{L P}$ & K1 & 162397 & MA_10434007_77578 & $\mathrm{C} / \mathrm{T}$ & Upstream gene variant & 0.892 & $1.14 \%$ \\
\hline \multirow[t]{3}{*}{ EP/LP } & Intercept & 51657 & MA_80954_29644 & $\mathrm{G} / \mathrm{A}$ & Downstream variant & 0.63 & $0.81 \%$ \\
\hline & & 60787 & MA_98424_947 & $\mathrm{C} / \mathrm{T}$ & Intron variant & 0.655 & $1.80 \%$ \\
\hline & & 123639 & MA_8790100_1384 & $\mathrm{A} / \mathrm{C}$ & Upstream variant & 0.628 & $0.75 \%$ \\
\hline
\end{tabular}




\begin{tabular}{|c|c|c|c|c|c|c|c|}
\hline & K1 & $59480 / 36496$ & MA_96191_7122 & $\mathrm{A} / \mathrm{G}$ & Synonymous & 0.6 & $2.37 \%$ \\
\hline & $\mathrm{K} 2$ & 117333 & MA_1045136_4310 & $\mathrm{T} / \mathrm{C}$ & Missense variant & 0.523 & $1.34 \%$ \\
\hline & $\mathrm{K} 3$ & 72414 & MA_122136_11653 & $\mathrm{A} / \mathrm{T}$ & Non-Coding & 0.617 & $4.05 \%$ \\
\hline Mass & Intercept & 166235 & MA_10435002_4986 & $\mathrm{G} / \mathrm{A}$ & Intergenic variant & 0.533 & $0.65 \%$ \\
\hline Index & Slope & 61096 & MA_99004_17108 & $\mathrm{G} / \mathrm{A}$ & Synonymous variant & 0.66 & $0.01 \%$ \\
\hline (Growth $\mathbf{x}$ & & 67181 & MA_109804_10278 & G/A & Missense variant & 0.612 & $0.05 \%$ \\
\hline \multirow[t]{4}{*}{ Density) } & & 1401 & MA_1378_4718 & $\mathrm{C} / \mathrm{A}$ & Exon/stop gained & 0.588 & $1.19 \%$ \\
\hline & & 138744 & MA_10427214_13968 & $\mathrm{G} / \mathrm{T}$ & Missense variant & 0.58 & $1.80 \%$ \\
\hline & & 162397 & MA_10434007_77578 & $\mathrm{C} / \mathrm{T}$ & Upstream variant & 0.627 & $1.44 \%$ \\
\hline & K1 & 21924 & MA_19222_1789 & $\mathrm{A} / \mathrm{G}$ & Upstream variant & 0.71 & $1.82 \%$ \\
\hline
\end{tabular}

*SNP: The SNP name was composed of the contig (MA_number) and SNP position on contig. For example, the first SNP MA_1043540_13733 
Several QTLs shared within each trait and across traits were observed in the analysis.

424 WD, RW, TRW and LNC had one (30469), two (165481 and 23808), one (111057) and one

425 (143628) QTL shared by two latent traits, respectively. One of the common QTL (30469) for

426 WD had a frequency of 0.72 with an $H^{2}{ }_{Q T L}$ of $4.50 \%$ for the slope trait, which indicates that it

427 is highly significant for the phenotypes. Common QTLs within RW were observed for slope,

$428 \mathrm{~K} 1$ and $\mathrm{K} 2$ latent traits, with moderate frequencies ranging from 0.521 to 0.615 and

429 influenced their respective traits to modest degree $\left(H^{2}{ }_{Q T L}\right.$ in ranges of $\left.0.18-2.66 \%\right)$.

430 For QTLs common across the different latent traits, QTL 165481 was shared between

431 LWD, RW and MOE; this is not surprising because of the close correlation between MOE

432 and wood density, which in turn generally show negative correlation to RW. Intron variant

433 MA_10434805g0010_165481 explained between $0.18-2.66 \%$ of the $H^{2}$ QTL observed in the

434 respective traits. The SNP associated with this QTL also had high frequencies of 0.602 and

4350.615 in MOE and RW explaining $H_{Q T L}^{2}$ of 1.00 and $2.66 \%$, respectively. It was also

436 observed that the SNP MA_10434805g0010_165481 is a common QTL within the wood

437 traits related to Width (Table 2). SNP MA_10435406g0010_167610 was shared between

438 WD, EWD and ENC. This SNP was characterized by having high frequencies in WD (0.71)

439 and ENC (0.685), however it had a moderate frequency of 0.545 for EWD. This QTL was

440 detected by the intercept latent trait for WD and EWD, and the slope latent trait in ENC

441 (Table 2), with $H^{2}$ QTL ranging from 0.01-4.64\%. The QTL had a high influence on the density

442 related traits as it explained $4.64 \%$ (WD) and 3.38\% (EWD).

443 Trees showing a positive correlation between growth and density had seven QTL

444 specific for this observed phenomenon (MI) and had modest influence on the trait $\left(H^{2}{ }_{Q T L}\right.$ in

445 the ranges $0.05-1.82 \%$ ). Five of the QTL were detected using the slope as the latent trait with

446 high frequencies ranging between 0.58 to 0.66 for SNP 61096 (Table 2). 

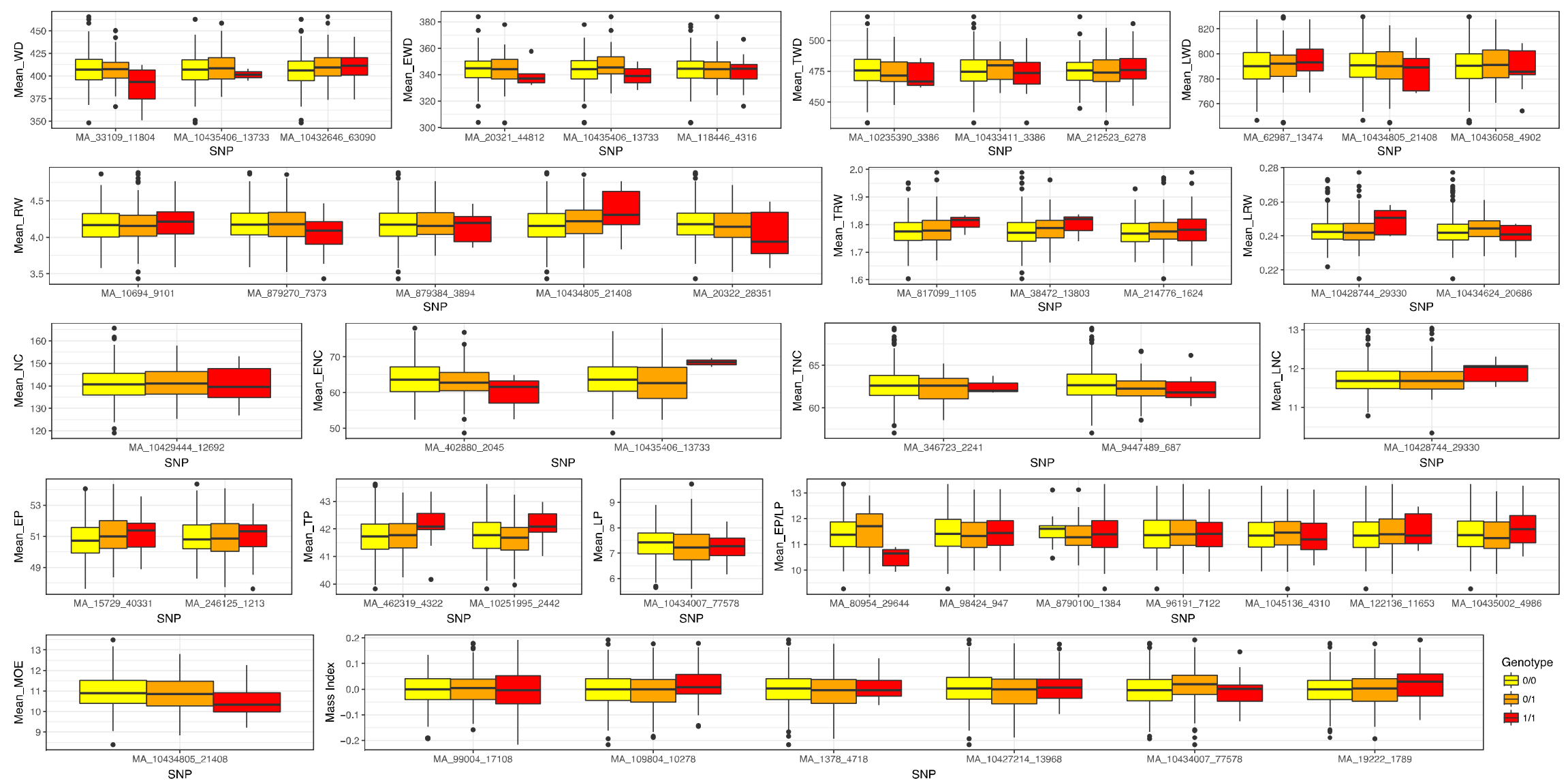

Genotyp 官 $0 / 1$

Fig 5. Box plot of the estimated genotypic effect on the phenotypes in the study. The significant SNPs associated and each one of the traits have 
Genetic association with phenotypes

454 Sequence capture and the SNP-trait associations allowed the mining of candidate genes 455 involved in spruce wood formation coupled with the identification of orthologous annotations and descriptions from Populus and Arabidopsis. This also allowed for the anchoring of significant markers on to the genetic linkage map for Norway spruce Fig 5.

RW, TRW, and LRW were associated with nine gene models. For RW five genes, endoglucanase 11-like, Alpha-dioxygenase 1 (DIOX1), Proliferating cell nuclear antigen 460 (PCNA), B3-DNA-binding and E3 ubiquitin-protein ligase were identified. The SNP MA_879270g0010_112391, a splice region variant, explained $2.56 \%$ of the $H^{2}$ QTL and is associated with DIOX1. Marker MA_20322g0010_23808 for RW is associated with the 463 protein domain for a plant specific B3-DNA binding protein, explaining $1.78 \%$ variation, with 464 similar orthologous genes in Arabidposis and Populus (Table S1). The three putative genes 465 associated with TRW are a Serine/threonine-protein kinase, a Homeodomain protein (HB2) 466 and a Senescence-associated protein, and all have high $H^{2}{ }_{Q T L}$ ranging from 2.14 to $4.50 \%$. 467 Contig MA_10434624 is homologos to a Pectin esterase and was associated with the downstream variant MA_10434624g0010_164772 for LRW. This may suggest a link between LRW and pectin modification. QTL associated with gene MA_214776g0010 for the TRW may be linked with serine/threonine-protein kinase gene (Os01g0689900), this occurrence of

471 kinase-like related genes was also observed across TRW, NC, EP, EP/LP and EWD (Table 472 S1). 
476 associated with these traits were either downstream or upstream of coding regions and may

477 thus act as modifiers of gene expression. The SNP MA_402880g0010_98508 (an upstream

478 gene variant) significantly associated with ENC located on gene MA_402880g0010 is

479 homologous to a Populus sphingolipid biosynthesis protein. SNP

480 MA_9447489g0010_126785 associated with TNC was located in the gene

481 MA_9447489g0010 which is homologous to a peptidase domain from Arabidopsis and

482 showed the highest $H_{Q T L}^{2}$ in the dataset (4.93\%). This domain is similar to an orthologous

483 zinc carboxypeptidase enzyme of Oryza sativa (Zn-dependent exopeptidases superfamily

484 protein) (Table S1).

485 Wood percentage traits, EP, LP, TP and the ratio of EP/LP had significant associations

486 with ten SNPs. Four of the six significant SNP variants for EP/LP are modifiers with the other

487 two SNPs, being a synonymous (MA_96191g0010_59480) and missense

488 (MA_1045136g0010_117333) variant. The synonymous SNP MA_96191g0010_59480 was

489 associated with the gene model MA_96191g0010, which is homologous to a $P$. sitchensis

490 Glycosyltransferase (GT), similar to UDP-glucosyltransferase 73B2 (AT4G34135) from

491 Arabidopsis. Five protein domains were also detected, that were linked to phytochrome

492 kinase substrate 1, TIR/NBS/LRR and zein binding domains (Table S1). The significant SNP

493 MA_15729g0010_16868, an intron variant, that is associated with EP, is located in the gene

494 MA_15729g0010, which is homologous to a DNA-3-methyladenine glycosylase II enzyme.

495 The SNPs identified for TP and LP are all downstream gene variants (Table 2).

496 WD, EWD, TWD and LWD had a total of 12 significant associations. For the

497 associations with WD we identified the SNP MA_10435406g0010_167610 that is a 3'-gene

498 variant which explained the highest $H_{Q T L}^{2}$ observed (4.64\%) and is located in a gene that is

499 homologous to a Phosphoadenosine phosphosulfate reductase gene cysH_2. This locus was

500 also detected for EWD and ENC explaining $H^{2}$ QTL of $3.38 \%$ and $0.01 \%$, respectively. A 
501 missense SNP, MA_33109g0010_30469, was associated with WD and located within the

502 gene MA_33109g0010 homologous to an Arabidopsis senescence associated gene 24 (Table

503 S1). The three significant SNPs identified for EWD were all modifiers, upstream and

504 downstream gene variants. Of the three significant SNP associations for TWD, two, SNP

505 MA_10235390_131698 (stop gained) and SNP MA_212523g0010_89044 (upstream gene

506 variant), were identified within genes. The intron variant MA_10433411g0010_160208

507 associated with TWD and is found in the gene MA_10433411g0010 that is homologous to an

508 Arabidopsis Transducin/WD40 repeat-like superfamily protein. Two of the three significant

509 SNPs identified for LWD were intron variants (MA_10434805g0010_165481 and

510 MA_10436058g0010_171223) with the third being a missense variant

511 (MA_62987g0010_43797). The SNP MA_10434805g0010_165481 was found in the gene

512 MA_10434805g0010, which is homologous to an Arabidopsis Proliferating Cell Nuclear

513 Antigen Protein (PCNA). This SNP is also associated with RW and explained 1.21\% and

$5142.66 \% H^{2}$ QTL, respectively.

515 The Mass Index trait, that is linked to a positive effect of wood volume growth and

516 increased density (growth $\mathrm{x}$ density) yielded a total of seven associated SNPs, with two

517 upstream gene variants, two missense variants one intergenic variant, one stop gained variant

518 and one synonymous nucleotide replacement (Table 2). The slope latent trait had five genes

519 with modest influence on the phenotype ranging from $0.01-1.80 \%$. The genes were

520 homologous to Arabidopsis GRAS transcription factor, Aluminium induced protein, Protein

521 virilizer, ARM repeat superfamily protein and an uncharacterized protein. The SNP

522 MA_1378g0010_1401 encodes for a premature stop codon (stop gained, a high impact

523 variant) on gene MA_1378g0010, which is homologous to an Arabidopsis protein virilizer

524 involved in mRNA splicing regulation. The gene homologous to the GRAS transcription

525 factor was associated with the SNP MA_99004g0010_61096 (a synonymous variant). The 
526 SNP MA_19222g0010_21921, an upstream gene variant, was located in the gene

527 MA_19222g0010 which is homologous to a Picea sitchensis ADP (NB-ARC domain) and 528 explained the highest $H_{Q T L}^{2}$ of $1.82 \%$ (Table S1).

529 Wood density traits were associated with a total of 12 genes, the largest number of 530 genes identified from the contigs. Percentage of wood was linked to ten putative genes, cell

531 width had nine putative genes and number of cells was associated with six genes. Two genes

532 were shared across multiple traits, PCNA was common across RW and LWD, and 533 phophoadenosine phosphosulfate reductase was shared across WD, EWD and ENC. Genes

534 with the Serine/threonine-protein phosphatase and TIR-NBS-LRR domains were also 535 identified across width, wood density and cell percentage traits. 


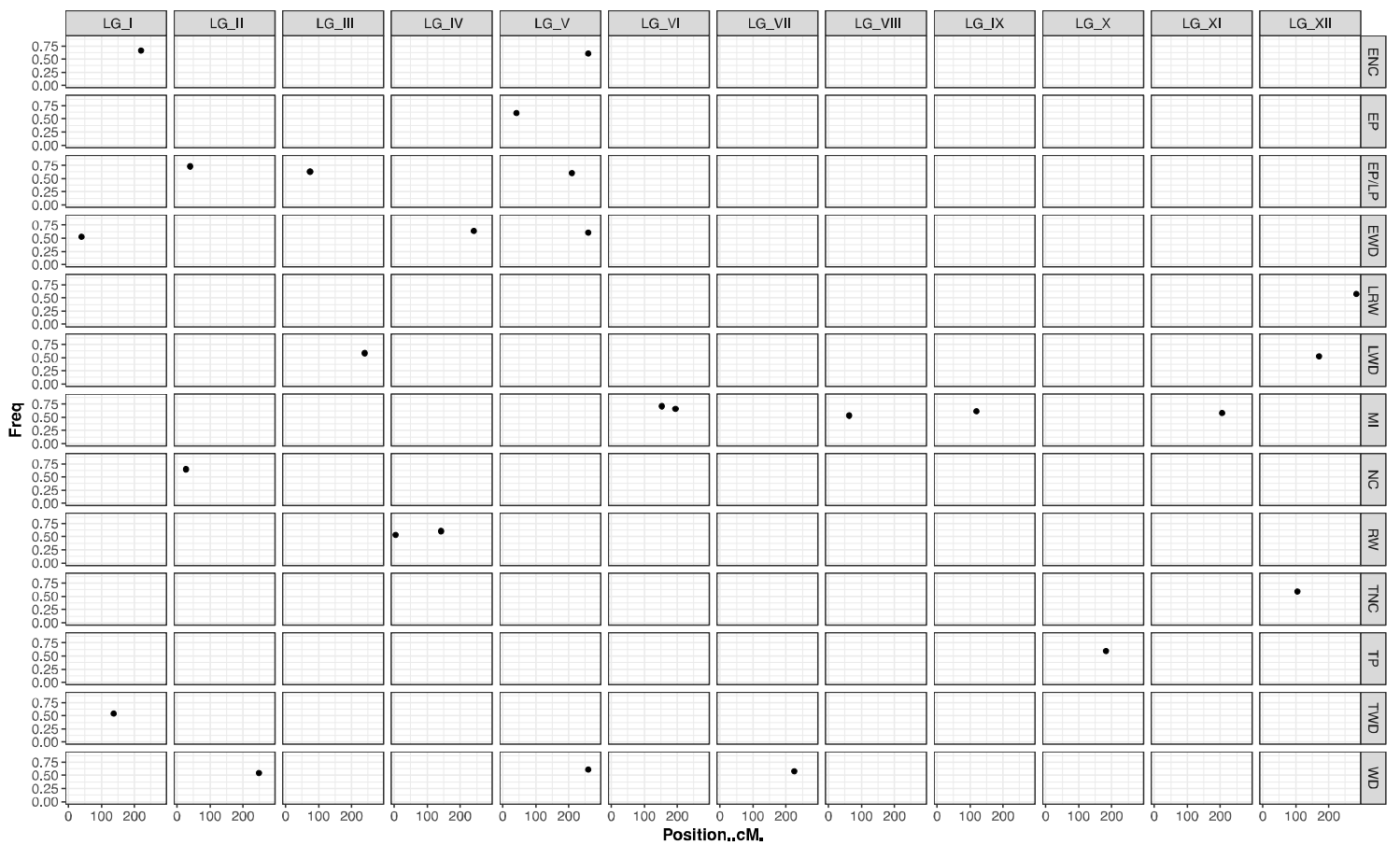

538 Fig 6. Frequencies of the significant markers selected using the multi-locus LASSO model for

539 whole ring, earlywood and latewood associated with contigs plotted against their locations on

540 a genetic linkage map derived from similar sequence captured probes. Significant associations

541 for the traits were identified on the twelve linkage groups (LG) as follows: [LG_I: EWD,

542 TWD and ENC], [LG_II: NC, EP/LP and WD], [LG_III: EP/LP and RW], [LG_IV: RW

543 and EWD], [LG_V: EP, EP/LP, ENC, EWD and WD], [LG_VI: MI], [LG_VII: WD],

544 [LG_VIII: MI], [LG_IX: MI], [LG_X: TP], [LG_XI: MI] and [LG_XII: TNC, LWD and 545 LRW].

\section{Discussion}

548 We applied a functional mapping approach in a genome-wide association mapping context 549 and identified 51 significant QTLs that were associated with wood formation in Norway 550 spruce. Previous work utilizing a functional mapping analysis in forest trees have used a 551 limited number of molecular markers (Li et al., 2014; Ma et al., 2002). Li et al., applied this 
552 analysis in a bi-parental Scots pine ( $P$. sylvestris L.) cross using 319 markers. Hence, our

553 work represents a major advance in that we have been able to apply this approach at a 554 genome-wide scale (178101 SNPs) on unrelated mother trees, with a dynamic functional trait 555 dataset comprising 15-time points/annual growth rings (i.e., cambial age). Latent traits 556 represent significant time points in the trait development allowing us to detect putative genes 557 at these critical junctures in wood formation.

558 The number of detected QTLs is relatively small compared with several recent studies 559 in Populus (Evans et al., 2014; McKown et al., 2014; Porth et al., 2013). The sample size, 560 number of SNPs used and the stringency with which we accepted significant SNPs 561 contributed to the modest number of QTL. Previous functional mapping studies, (Li et al., 562 2014) involving SNPs in conifers have used two levels of evaluating QTLs, whereby they 563 have suggestive and significant QTL. In our study, we only reported significant QTL. As

564 indicated in Hall et al (2016), there should be hundreds to thousands of QTL of moderate to 565 very small effect related to growth and wood quality traits in trees. Hence, a large population 566 and accurate phenotyping are required for a reliable identification of most QTLs (Korte \& 567 Farlow, 2013). However, the sample size of our study allowed the detection of the 568 largest/most significant QTL. The study identified significant associations explaining 569 relatively small proportions of the phenotypic variance being observed, ranging from 0.01$5704.93 \%$. This is in line with other studies of QTL for wood traits (González-Martínez et al., 571 2007; Porth et al., 2013).

572

Genetic associations with potential to improve wood properties

574 With all the SNPs, having been derived from known genomic positions, it was 575 possible to identify genes linked to the associated QTLs and infer their potential function in 576 wood formation. 
The gene MA_10694g0010 is homologous to an enzyme involved in cell wall

578 biosynthesis, endoglucanase 11-like, and was associated with RW (intercept latent) (Table

579 S1). The association of this gene with the RW intercept implies that the gene influences the

580 trait throughout the growth period. This enzyme is a vital component of xylogenesis and is

581 involved in the active digestion of the primary cell wall (Goulao et al., 2011). The

582 endoglucanase 11-like, was associated with a synonymous SNP MA_10694g0010_11535 for

583 (RW) suggesting an involvement in cell expansion and cell wall loosening during wood

584 formation. Endoglucanases have been proposed as enzymes involved in controlling cell wall

585 loosening (Cosgrove, 2005). Endoglucanase 11-like gene is part of the endo-1 family in

586 which the eno-1-4- $\beta$-glucanase Korrigan gene belongs. Characterisation of the Korrigan gene

587 in $P$. glauca has identified it to be functionally conserved and essential for cellulose synthesis

588 (Maloney et al., 2012). Hence, MA_10694g0010 is a candidate for the remodelling of cell

589 walls that affects the mechanical and growth properties of wood cells, and consequently

590 annual ring width.

591 The synonymous SNP MA_20322g0010_23808 is associated with RW located on

592 gene MA_20322g0010 which is homologous with a plant specific B3-DNA binding protein

593 domain, that is shared among various plant-specific transcription factors. This includes

594 transcription factors involved in auxin and abscisic acid responsive transcription (Yamasaki et

595 al., 2004). Auxin is one of the central phytohormones in the control of plant growth and

596 development (Abel \& Theologis, 1996), and also known to be involved in cell wall loosening

597 and elongation (Cosgrove, 2016). This suggests a possible functional role for

598 MA_20322g0010 in influencing RW.

599 An intron variant located in the MA_10434805g0020 gene, which is homologous to

600 PCNA was detected across several phenotypes (LWD, RW and MOE) associated with the

601 slope latent trait (Table 2). The detection of this gene across these phenotypes using the slope 
602 latent trait implies that the gene affects the rate of change of these phenotypes. Thus, this

603 would be a good gene to target for further studies. PCNA proteins function as integral

604 enzymes in the regulatory pathways of cell cycle regulation and DNA metabolism (Maga \&

605 Hübscher, 2003). PCNA has been associated with chromatin remodelling, DNA repair, sister-

606 chromatid cohesion and cell cycle control, which are all vital processes in plant growth

607 (Strzalka \& Ziemienowicz, 2010), but it has not been previously associated with wood 608 formation traits.

609 In our study we detected a significant downstream SNP 610 (MA_10434624g0010_164772) associated with LRW on gene MA_10434624g0020,

611 homologous to pectinmethylesterases (PMEs), which are cell wall associated enzymes

612 responsible for demethylation of polygalacturonans (Phan et al., 2007). This enzyme has been

613 shown to be linked with many developmental processes in plants, such as, cellular adhesion

614 and stem elongation (Micheli, 2001). An association study in White spruce identified a

615 significant nonsynonymous SNP coding for cysteine associated with earlywood and total

616 wood cell wall thickness associated with pectinmethylesterase (Beaulieu et al., 2011). Our

617 study identified a PME SNP association in the latewood stage, supporting the importance of

618 PMEs in wood cell development.

619 A SNP (MA_10435406g0010_167910) downstream on gene MA_10435406g0010

620 was detected across the traits ENC, WD and EWD. The association of this gene with the WD

621 and EWD intercept implies that it an impact on the overall development of density throughout

622 the growth period. Since density is correlated with number of cells, this association with the

623 slope latent trait of ENC means the gene influences its rate of change. The gene is

624 homologous to Phosphoadenosine phosphosulfate reductase (PAPS), which plays a central

625 role in the reduction of sulphur in plants. An analysis of PAPS enzymes in Arabidopsis (Klein

626 \& Papenbrock, 2004) and Populus (Kopriva et al., 2004) revealed that enzymes involved in 
627 sulphate-conjugation, play an important role in plant growth and development (Klein \&

628 Papenbrock, 2004). Reduced sulphur is utilized by the sulphate assimilation pathway for the 629 synthesis of essential amino acids cysteine and methionine (Kopriva \& Koprivova, 2004).

630 Methione acts as a methyl donor in both lignin, hemicellulose and pectin biosynthesis

631 providing a possible mechanism of how PAPS could influence wood density and number of 632 cells.

633 When analyzing QTLs detected for traits linked to the percentage of cells (EP, LP and 634 EP/LP) we identified three putative candidate genes, DNA-3-methyladenine glycosylase II 635 enzyme, phytochrome kinase substrate 1 and glycosyltransferase. DNA-3-methyladenine 636 glycosylase II enzyme is responsible for carrying out base excision repairs (BER) in the 637 genome in order to maintain genomic integrity. This enzyme has the ability to initiate a broad 638 substrate recognition and provides a wide resistance to DNA damaging agents (Wyatt et al., 639 1999). This DNA repair capacity can be expected to be essential for the process of cell 640 propagation and growth.

641 A synonymous SNP (MA_96191_59480) within the gene MA_96191g0010, which is 642 homologous to Glucosyltransferase in P. sitchensis was associated with EP/LP. Glycosyl 643 transferases operate by facilitating the catalytic sequential transfer of sugars from activated 644 donors to acceptor molecules that form region and stereospecific glycosidic linkages (Lairson 645 et al., 2008). The Arabidopsis ortholog (UDP-glucosyltransferase 73B2) encodes for a 646 putative flavonol 7-O-glucosyltransferase involved in stress responses. In our study, this 647 significant association was associated with EP/LP, however a nonsynonymous variant in a 648 gene coding for a Glycosyl transferase in Populus was associated with fibre development and 649 elongation (Porth et al., 2013). Therefore, gene MA_96191g0010 is a novel candidate for 650 further investigation of how flavonol metabolism may influence the proportion of early and 651 late wood in Norway spruce. 
652 Two genes concerning wood formation, PAPS and PCNA, were also detected across

653 related traits density, growth number of cells and MOE. Significant SNP

654 (MA_10435406_167610) in the PAPS reductase gene is common across ENC, WD and

655 EWD, with SNP MA_10434805_165481 located in an intron for a gene encoding for PCNA

656 protein being detected across WD, RW and MOE (Table S1). The presence of these common

657 QTL suggests that these traits might be under the control of the same genes or genetic

658 pathways. Chen et al (2014) reported a significant positive genetic correlation between wood

659 density and MOE, which increased with tree age. However, wood volume growth and density

660 have a negative correlation (Chen et al., 2014), with our study being the first to detect QTLs

661 for trees exhibiting a positive correlation for this phenomenon (MI). The common QTL

662 observed across WD, EWD and ENC indicates that the number of cells during the juvenile

663 wood development stages has a significant impact on the overall density. The seasonal

664 changes in EWD to LWD has been speculated to be due to a change in auxin levels leading to

665 the initiation of wall-thickening phase, which has a direct impact on the wood quality traits

666 such as MOE. This phase coincides with the cessation of height growth and where available

667 resources are used for cell-wall thickening (Sewell et al., 2000), which may explain the

668 common QTL between LWD, RW and MOE, as part of the same feedback loop mechanism.

669 We identified two associations to homologous genes related to nucleic acid repair

670 functions, DICER-LIKE3 (DCL3) and DNA mismatch repair protein (MSH5), which are

671 concerned with RNA processing as well as DNA repair, respectively. These genes are

672 involved in ensuring the fidelity of DNA replication and to preserve genomic integrity (Hsieh

$673 \&$ Yamane, 2008). These genes are possibly associated with cambial cell division and endo-

674 reduplication during wood formation and can conceivably have effects on wood density.

675 An association for TWD with a SNP located upstream of gene MA_212523g0010, is

676 homologous to Kinesin-related protein 13 (gene-L484_021891). Kinesin-related proteins are 
677 known to be involved in secondary wall deposition, which can impact wood density (Zhong et

$678 a l ., 2002)$, cell wall strength and oriented deposition of cellulose microfibrils.

679 Several receptor-like Kinases (TIR/NBS/LRR and Serine/threonine-protein

680 phosphatase) homologs were identified across traits (TRW, NC, EP, EP/LP and EWD) (Table

681 S1). These protein domains control a large range of processes including hormone perception

682 and plant development. Approximately $2.5 \%$ of the annotated genes in Arabidopsis genome

683 are RLK homologs (Shiu \& Bleecker, 2001), where they among other functions play an

684 important role in the differentiation and separation of xylem and phloem cells (Fisher \&

685 Turner, 2007). Similar to our study a synonymous SNP in a RLK gene was associated with

686 early wood proportion (EP) in White spruce (Beaulieu et al., 2011), hence RLKs seem to be

687 involved in modifying a number of different wood properties from density to cell identity and

688 number.

689 Norway spruce trees that possess the ability of fast growth and high wood density are 690 very rare, but such trees and associated SNPs were discovered in our study. Trees combining

691 these traits are of high interest to forest industries and owners, and thus also in focus for 692 breeders. Of the seven genes significantly linked to this phenomenon of particular interest was 693 a synonymous SNP on MA_99004g0100 gene homologous to a transcription factor from the 694 GRAS family (Table S1). GRAS is an important class of plant-specific proteins derived from 695 three members: GIBBERELLIC-ACID INSENSITIVE (GAI), REPRESSOR of GAI (RGA) 696 and SCARECROW (SCR) (GRAS) (Hirsch \& Oldroyd, 2009). GRAS genes are known to be 697 involved in the regulation of plant development through the regulation of gibberellic acid 698 (GA) and light signalling (Cenci \& Rouard, 2017; Hirsch \& Oldroyd, 2009). Furthermore GA 699 signalling has also been shown to stimulate wood formation in Populus (Mauriat \& Moritz, 700 2009). Thus, the GRAS transcription factor identified here and the other six genes positively 
701 associated with MI provide interesting genetic markers and tools to understand this

702 phenomenon.

703

704 Conclusion

705 This work has dissected the genetic basis of wood properties in Norway spruce with use of

706 functional association mapping. In total, we identified 51 Significant QTLs for wood

707 properties and mining of candidate genes located in the vicinity of significant QTLs identified

708 genes that could be directly or indirectly responsible for variations in the observed traits.

709 Significant novelty in our results is provided by the identification of QTLs associated to both

710 high wood density and fast growth, thus larger biomass. These genes are candidates for

711 further functional verification in Norway spruce.

712

\section{Acknowledgements}

714 We acknowledge the support of the Bio4Energy research organization for wood property

715 analyses and evaluations. All genetic data was obtained through funding from the Knut and

716 Alice Wallenberg foundation. SSF project support of continuing this work. JB is supported

717 though a postdoc position funded by the Kempe foundation.

718 
Abel, S., \& Theologis, A. (1996). Early Genes and Auxin Action. Plant Physiology, 111(1), 9.

Beaulieu, J., Doerksen, T., Boyle, B., Clément, S., Deslauriers, M., Beauseigle, S., Blais, S., Poulin, P.-L., Lenz, P., Caron, S., Rigault, P., Bicho, P., Bousquet, J., \& MacKay, J. (2011). Association Genetics of Wood Physical Traits in the Conifer White Spruce and Relationships With Gene Expression. Genetics, 188(1), 197-214. doi: 10.1534/genetics.110.125781

Beavis, W. D. (1998). QTL analyses: power, precision, and accuracy. Molecular dissection of complex traits, 1998, 145-162.

Bernhardsson, C., Vidalis, A., Wang, X., Scofield, D. G., Shiffthaler, B., Baison, J., Street, N. R., Garcia Gil, M. R., \& Ingvarsson, P. K. (2018). An ultra-dense haploid genetic map for evaluating the highly fragmented genome assembly of Norway spruce (<em>Picea abies $</$ em>). bioRxiv. doi: 10.1101/292151

Bertaud, F., \& Holmbom, B. (2004). Chemical composition of earlywood and latewood in Norway spruce heartwood, sapwood and transition zone wood. Wood Science and Technology, 38(4), 245-256. doi: 10.1007/s00226-004-0241-9

Bradbury, P. J., Zhang, Z., Kroon, D. E., Casstevens, T. M., Ramdoss, Y., \& Buckler, E. S. (2007). TASSEL: software for association mapping of complex traits in diverse samples. Bioinformatics, 23(19), 2633-2635.

Cenci, A., \& Rouard, M. (2017). Evolutionary Analyses of GRAS Transcription Factors in Angiosperms. Frontiers in Plant Science, 8, 273. doi: 10.3389/fpls.2017.00273

Chen, Z.-Q., Gil, M. R. G., Karlsson, B., Lundqvist, S.-O., Olsson, L., \& Wu, H. X. (2014). Inheritance of growth and solid wood quality traits in a large Norway spruce population tested at two locations in southern Sweden. Tree Genetics \& Genomes, 10(5), 1291-1303.

Cingolani, P., Platts, A., Wang, L. L., Coon, M., Nguyen, T., Wang, L., Land, S. J., Lu, X., \& Ruden, D. M. (2012). A program for annotating and predicting the effects of single nucleotide polymorphisms, SnpEff: SNPs in the genome of Drosophila melanogaster strain w1118; iso-2; iso-3. Fly, 6(2), 80-92.

Cosgrove, D. J. (2005). Growth of the plant cell wall. Nat Rev Mol Cell Biol, 6(11), 850-861. doi: http://www.nature.com/nrm/journal/v6/n11/suppinfo/nrm1746 S1.html

Cosgrove, D. J. (2016). Catalysts of plant cell wall loosening. F1000Research, 5, F1000 Faculty Rev-1119. doi: 10.12688/f1000research.7180.1

Danecek, P., Auton, A., Abecasis, G., Albers, C. A., Banks, E., DePristo, M. A., Handsaker, R. E., Lunter, G., Marth, G. T., \& Sherry, S. T. (2011). The variant call format and VCFtools. Bioinformatics, 27(15), 2156-2158.

Dutilleul, P., Herman, M., \& Avella-Shaw, T. (1998). Growth rate effects on correlations among ring width, wood density, and mean tracheid length in Norway spruce (Picea abies). Canadian Journal of Forest Research, 28(1), 56-68.

Evans, L. M., Slavov, G. T., Rodgers-Melnick, E., Martin, J., Ranjan, P., Muchero, W., Brunner, A. M., Schackwitz, W., Gunter, L., Chen, J.-G., Tuskan, G. A., \& DiFazio, S. P. (2014). Population genomics of Populus trichocarpa identifies signatures of selection and adaptive trait associations. Nat Genet, 46(10), 1089-1096. doi: 10.1038/ng.3075

http://www.nature.com/ng/journal/v46/n10/abs/ng.3075.html\#supplementary-information

Evans, R. (1994) Rapid Measurement of the Transverse Dimensions of Tracheids in Radial Wood Sections from Pinus Radiata, Holzforschung 48: 168-72. 
767 Evans, R. (2006) Wood stiffness by X-ray diffractometry. In: Stokke DD, Groom HL (eds) Characterization of the cellulosic cell wall. Wiley, Hoboken, pp. 138-146.

Fisher, K., \& Turner, S. (2007). PXY, a receptor-like kinase essential for maintaining polarity during plant vascular-tissue development. Current Biology, 17(12), 1061-1066.

Gao, H., Wu, Y., Li, J., Li, H., Li, J., \& Yang, R. (2014). Forward LASSO analysis for highorder interactions in genome-wide association study. Briefings in Bioinformatics, 15(4), 552-561.

Gilmour, A., Gogel, B., Cullis, B., Welham, S., Thompson, R., Butler, D., Cherry, M., Collins, D., Dutkowski, G., \& Harding, S. (2014). ASReml user guide. Release 4.1 structural specification. VSN International Ltd, Hemel Hempstead, HP1 1ES, UK www. vsni. co. $u k$.

González-Martínez, S. C., Wheeler, N. C., Ersoz, E., Nelson, C. D., \& Neale, D. B. (2007). Association genetics in Pinus taeda LI Wood property traits. Genetics, 175(1), 399409.

Goulao, L. F., Vieira-Silva, S., \& Jackson, P. A. (2011). Association of hemicellulose- and pectin-modifying gene expression with Eucalyptus globulus secondary growth. Plant Physiology and Biochemistry, 49(8), 873-881. doi: https://doi.org/10.1016/j.plaphy.2011.02.020

Hallingbäck, H. R., Sánchez, L., \& Wu, H. X. (2014). Single versus subdivided population strategies in breeding against an adverse genetic correlation. Tree Genetics \& Genomes, 10(3), 605-617.

Hannrup, B., Cahalan, C., Chantre, G., Grabner, M., Karlsson, B., Bayon, I. L., Jones, G. L., Müller, U., Pereira, H., \& Rodrigues, J. C. (2004). Genetic parameters of growth and wood quality traits in Picea abies. Scandinavian Journal of Forest Research, 19(1), 14-29.

Hauksson, J. B., Bergqvist, G., Bergsten, U., Sjöström, M., \& Edlund, U. (2001). Prediction of basic wood properties for Norway spruce. Interpretation of Near Infrared Spectroscopy data using partial least squares regression. Wood Science and Technology, 35(6), 475-485. doi: 10.1007/s00226-001-0123-3

Heuven, H. C., \& Janss, L. L. (2010). Bayesian multi-QTL mapping for growth curve parameters. Paper presented at the BMC proceedings.

Hill, W., \& Weir, B. (1988). Variances and covariances of squared linkage disequilibria in finite populations. Theoretical population biology, 33(1), 54-78.

Hirsch, C. D., Evans, J., Buell, C. R., \& Hirsch, C. N. (2014). Reduced representation approaches to interrogate genome diversity in large repetitive plant genomes. Briefings in Functional Genomics, elt051.

Hirsch, S., \& Oldroyd, G. E. D. (2009). GRAS-domain transcription factors that regulate plant development. Plant Signaling \& Behavior, 4(8), 698-700.

Hsieh, P., \& Yamane, K. (2008). DNA mismatch repair: molecular mechanism, cancer, and ageing. Mechanisms of ageing and development, 129(7), 391-407.

Huang, X., \& Han, B. (2014). Natural variations and genome-wide association studies in crop plants. Annual review of plant biology, 65, 531-551.

Klein, M., \& Papenbrock, J. (2004). The multi-protein family of Arabidopsis sulphotransferases and their relatives in other plant species. Journal of experimental botany, 55(404), 1809-1820. doi: 10.1093/jxb/erh183

Kopriva, S., Hartmann, T., Massaro, G., Hönicke, P., \& Rennenberg, H. (2004). Regulation of sulfate assimilation by nitrogen and sulfur nutrition in poplar trees. Trees, 18(3), 320326. doi: 10.1007/s00468-003-0309-4 
815 Kopriva, S., \& Koprivova, A. (2004). Plant adenosine 5'-phosphosulphate reductase: the past,

816

817

818

819

820

821

822

823

824

825

826

827

828

829

830

831

832

833

834

835

836

837

838

839

840

841

842

843

844

845

846

847

848

849

850

851

852

853

854

855

856

857

858

859

860

861

862

863

864 the present, and the future. Journal of experimental botany, 55(404), 1775-1783. doi: $10.1093 / \mathrm{jxb} / \mathrm{erh} 185$

Korte, A., \& Farlow, A. (2013). The advantages and limitations of trait analysis with GWAS: a review. Plant methods, 9(1), 1.

Lairson, L., Henrissat, B., Davies, G., \& Withers, S. (2008). Glycosyltransferases: structures, functions, and mechanisms. Annu. Rev. Biochem., 77, 521-555.

Lande, R., \& Thompson, R. (1990). Efficiency of marker-assisted selection in the improvement of quantitative traits. Genetics, 124(3), 743-756.

Langmead, B., \& Salzberg, S. L. (2012). Fast gapped-read alignment with Bowtie 2. Nature Methods, 9(4), 357-359.

Li, H., \& Durbin, R. (2009). Fast and accurate short read alignment with Burrows-Wheeler transform. Bioinformatics, 25(14), 1754-1760.

Li, H., Handsaker, B., Wysoker, A., Fennell, T., Ruan, J., Homer, N., Marth, G., Abecasis, G., \& Durbin, R. (2009). The sequence alignment/map format and SAMtools. Bioinformatics, 25(16), 2078-2079.

Li, Z., Hallingbäck, H. R., Abrahamsson, S., Fries, A., Gull, B. A., Sillanpää, M. J., \& GarcíaGil, M. R. (2014). Functional multi-locus QTL mapping of temporal trends in Scots pine wood traits. G3: Genes| Genomes| Genetics, 4(12), 2365-2379.

Li, Z., \& Sillanpää, M. J. (2015). Dynamic Quantitative Trait Locus Analysis of Plant Phenomic Data. Trends in Plant Science, 20(12), 822-833. doi: http://dx.doi.org/10.1016/j.tplants.2015.08.012

Ma, C.-X., Casella, G., \& Wu, R. (2002). Functional mapping of quantitative trait loci underlying the character process: a theoretical framework. Genetics, 161(4), 17511762.

Maga, G., \& Hübscher, U. (2003). Proliferating cell nuclear antigen (PCNA): a dancer with many partners. Journal of Cell Science, 116(15), 3051.

Maloney, V. J., Samuels, A. L., \& Mansfield, S. D. (2012). The endo-1, 4- $\beta$-glucanase Korrigan exhibits functional conservation between gymnosperms and angiosperms and is required for proper cell wall formation in gymnosperms. New Phytologist, 193(4), 1076-1087.

Mauriat, M., \& Moritz, T. (2009). Analyses of GA20ox-and GID1-over-expressing aspen suggest that gibberellins play two distinct roles in wood formation. The Plant Journal, 58(6), 989-1003.

McKenna, A., Hanna, M., Banks, E., Sivachenko, A., Cibulskis, K., Kernytsky, A., Garimella, K., Altshuler, D., Gabriel, S., \& Daly, M. (2010). The Genome Analysis Toolkit: a MapReduce framework for analyzing next-generation DNA sequencing data. Genome Research, 20(9), 1297-1303.

McKown, A. D., Klápště, J., Guy, R. D., Geraldes, A., Porth, I., Hannemann, J., Friedmann, M., Muchero, W., Tuskan, G. A., \& Ehlting, J. (2014). Genome-wide association implicates numerous genes underlying ecological trait variation in natural populations of Populus trichocarpa. New Phytologist, 203(2), 535-553.

Meinshausen, N., \& Bühlmann, P. (2010). Stability selection. Journal of the Royal Statistical Society: Series B (Statistical Methodology), 72(4), 417-473.

Micheli, F. (2001). Pectin methylesterases: cell wall enzymes with important roles in plant physiology. Trends in Plant Science, 6(9), 414-419. doi: http://doi.org/10.1016/S1360$\underline{1385(01) 02045-3}$

Moritsuka, E., Hisataka, Y., Tamura, M., Uchiyama, K., Watanabe, A., Tsumura, Y., \& Tachida, H. (2012). Extended linkage disequilibrium in noncoding regions in a conifer, Cryptomeria japonica. Genetics, 190(3), 1145-1148. 
865

866

867

868

869

870

871

872

873

874

875

876

877

878

879

880

881

882

883

884

885

886

887

888

889

890

891

892

893

894

895

896

897

898

899

900

901

902

903

904

905

906

907

908

909

910

911

912

913

914

Neale, D. B., \& Savolainen, O. (2004). Association genetics of complex traits in conifers. Trends in Plant Science, 9(7), 325-330.

Nystedt, B., Street, N. R., Wetterbom, A., Zuccolo, A., Lin, Y.-C., Scofield, D. G., Vezzi, F., Delhomme, N., Giacomello, S., \& Alexeyenko, A. (2013). The Norway spruce genome sequence and conifer genome evolution. Nature, 497(7451), 579-584.

Olesen, P. (1977). The variation of the basic density level and tracheid width within the juvenile and mature wood of Norway spruce. For. Tree Improv, 12, 1-22.

Parchman, T. L., Gompert, Z., Mudge, J., Schilkey, F. D., Benkman, C. W., \& Buerkle, C. (2012). Genome-wide association genetics of an adaptive trait in lodgepole pine. Molecular Ecology, 21(12), 2991-3005.

Phan, T. D., Bo, W., West, G., Lycett, G. W., \& Tucker, G. A. (2007). Silencing of the Major Salt-Dependent Isoform of Pectinesterase in Tomato Alters Fruit Softening. Plant Physiology, 144(4), 1960-1967. doi: 10.1104/pp.107.096347

Porth, I., Klapšte, J., Skyba, O., Hannemann, J., McKown, A. D., Guy, R. D., DiFazio, S. P., Muchero, W., Ranjan, P., \& Tuskan, G. A. (2013). Genome-wide association mapping for wood characteristics in Populus identifies an array of candidate single nucleotide polymorphisms. New Phytologist, 200(3), 710-726.

Resende, M. D., Resende, M. F., Sansaloni, C. P., Petroli, C. D., Missiaggia, A. A., Aguiar, A. M., Abad, J. M., Takahashi, E. K., Rosado, A. M., \& Faria, D. A. (2012). Genomic selection for growth and wood quality in Eucalyptus: capturing the missing heritability and accelerating breeding for complex traits in forest trees. New Phytologist, 194(1), 116-128.

Sewell, M., Bassoni, D., Megraw, R., Wheeler, N., \& Neale, D. (2000). Identification of QTLs influencing wood property traits in loblolly pine (Pinus taeda L.). I. Physical wood properties. TAG Theoretical and Applied Genetics, 101(8), 1273-1281.

Shiu, S.-H., \& Bleecker, A. B. (2001). Plant receptor-like kinase gene family: diversity, function, and signaling. Sci stke, 113(113), re22.

Strauss, S., Lande, R., \& Namkoong, G. (1992). Limitations of molecular-marker-aided selection in forest tree breeding. Canadian Journal of Forest Research, 22(7), 10501061.

Strzalka, W., \& Ziemienowicz, A. (2010). Proliferating cell nuclear antigen (PCNA): a key factor in DNA replication and cell cycle regulation. Annals of botany, 107(7), 11271140.

Team, R. (2015). RStudio: integrated development for R. RStudio, Inc., Boston, MA URL http://www. rstudio. com.

Thavamanikumar, S., Southerton, S. G., Bossinger, G., \& Thumma, B. R. (2013). Dissection of complex traits in forest trees-opportunities for marker-assisted selection. Tree Genetics \& Genomes, 9(3), 627-639.

Thumma, B. R., Southerton, S. G., Bell, J. C., Owen, J. V., Henery, M. L., \& Moran, G. F. (2010). Quantitative trait locus (QTL) analysis of wood quality traits in Eucalyptus nitens. Tree Genetics \& Genomes, 6(2), 305-317.

Tibshirani, R. (1996). Regression shrinkage and selection via the lasso. Journal of the Royal Statistical Society. Series B (Methodological), 267-288.

Vidalis, A., Scofield, D. G., Neves, L. G., Bernhardsson, C., García-Gil, M. R., \& Ingvarsson, P. (2018). Design and evaluation of a large sequence-capture probe set and associated SNPs for diploid and haploid samples of Norway spruce (Picea abies). bioRxiv. doi: $10.1101 / 291716$

Wyatt, M. D., Allan, J. M., Lau, A. Y., Ellenberger, T. E., \& Samson, L. D. (1999). 3methyladenine DNA glycosylases: structure, function, and biological importance. Bioessays, 21(8), 668-676. 
915 Yamasaki, K., Kigawa, T., Inoue, M., Tateno, M., Yamasaki, T., Yabuki, T., Aoki, M., Seki, 916 E., Matsuda, T., Tomo, Y., Hayami, N., Terada, T., Shirouzu, M., Osanai, T., Tanaka, 917 A., Seki, M., Shinozaki, K., \& Yokoyama, S. (2004). Solution Structure of the B3 918 DNA Binding Domain of the Arabidopsis Cold-Responsive Transcription Factor $919 \quad$ RAV1. The Plant Cell, 16(12), 3448.

920

921

922

Zhong, R., Burk, D. H., Morrison, W. H., \& Ye, Z.-H. (2002). A kinesin-like protein is essential for oriented deposition of cellulose microfibrils and cell wall strength. The Plant Cell, 14(12), 3101-3117. 